\title{
Time-frequency analysis of cardiovascular signals and their dynamic interactions.
}

\author{
M. Orini, P. Laguna, L.T. Mainardi, R. Bailón
}

February 20, 2017 


\section{Contents}

1 Time-frequency analysis of cardiovascular signals and their dynamic interactions. 3

1.1 Introduction . . . . . . . . . . . . . . . . . . . . 2

1.1.1 Spectral analysis and its limitations . . . . . . . . . . . . . . 2

1.2 Studying signals: Time-frequency representations . . . . . . . . . . . . . . . . . . 6

1.2.1 Introducing the Cohen's Class . . . . . . . . . . . . . . . . . . 6

1.2.2 Equivalent definitions of Cohen's class distributions . . . . . . . . . . . . . 6

1.2.3 The Wigner-Ville Distribution and the interference terms . . . . . . . . . . . 8

1.2.4 Time-frequency resolution . . . . . . . . . . . . . . . . . . 9

1.2.5 The smoothed-pseudo Wigner-Ville distribution (SPWVD) . . . . . . . . . . . 11

1.2.6 From time-frequency representations to time-frequency spectra . . . . . . . . . . . 13

1.3 Studying interactions: Multivariate time-frequency representations . . . . . . . . . . . . . 14

1.3.1 Time-frequency coherence . . . . . . . . . . . . . . . . . . 14

1.3.2 Time-frequency phase differences . . . . . . . . . . . . . . . . 16

1.3.3 Time-frequency phase locking . . . . . . . . . . . . . . . . . . 17

1.3.4 Time-frequency partial spectrum . . . . . . . . . . . . . . . . . 18

1.4 Example of analysis of cardiovascular signals . . . . . . . . . . . . . . . . . . . . 19

1.5 Final considerations . . . . . . . . . . . . . . . . . . . . . 20 
Chapter 1

Time-frequency analysis of cardiovascular signals and their dynamic interactions. 


\begin{abstract}
Cardiovascular signals are intrinsically non-stationary and interact through dynamic mechanisms to maintain blood pressure homeostasis in response to internal and external perturbations. The assessment of changes in cardiovascular signals and in their interactions provides valuable information regarding the cardiovascular function. Time-frequency analysis is a useful tool to study the time-varying nature of the cardiovascular system because it provides a joint representation of a signal in the temporal and spectral domain that allows to track the instantaneous frequency, amplitude and phase of non-stationary processes. The time-frequency distributions described in this chapter belong to the Cohen's class, and can be derived from the Wigner-Ville distribution, which represents the fundamental basis of this unified framework. Time-frequency analysis can be extended to the study of the dynamic interactions between two or more non-stationary processes. Time-frequency coherence, phase-delay, phase-locking and partialspectra are estimators that assess changes in the coupling strength and phase shift of signals generated by a complex system.

This chapter introduces the reader to multivariate time-frequency analysis and covers both theoretical and practical aspects. The application of these methodologies in the study of the dynamic interactions between the most important variables of the cardiovascular function is discussed. In the introduction, classical spectral analysis of cardiovascular signals is reviewed along with its physiological interpretation. The limitations of this framework provides a motivation for implementing non-stationary tools. In the first section, time-frequency representations based on the Wigner-Ville distribution are introduced and important aspects, such as the interference cross-terms and their elimination, the time and frequency resolution and the estimation of time-frequency spectra, are described. The second section describes algorithms to assess the dynamic interactions between non-stationary signals, including time-frequency coherence, phase delay and partial spectra, while the third section provides examples of multivariate time-frequency analysis of cardiovascular data recorded during a standard test to induce an autonomic response.
\end{abstract}




\subsection{Introduction}

A process is stationary if its probability distribution and therefore its main statistical properties do not change over time. There are different degrees and types of stationarity and a process can be stationary or non-stationary depending on the temporal scale of observation [1]. In the analysis of cardiovascular signals, stationarity is considered an exception rather than the rule. For example, the quantification of the degree of non-stationarity [1] of cardiovascular signals has demonstrated that heart rate variability is non-stationary even when recorded at rest [2]. Cardiovascular signals are intrinsically non-stationary and their interactions are time-varying because the system has to dynamically adjust to an ever-changing environment in order to maintain its function (blood pressure homeostasis). The quantification of changes and dynamic interactions within the cardiovascular system provides valuable information for understanding its function and recognizing possible dysfunctions.

The ultimate aim of most of signal processing methodologies is to provide a simple description of complex systems that captures the most relevant features for a given application. Over the years, two approaches have emerged to transform raw data into more useful representations. One includes model-based methodologies, where some a-priory knowledge is used to propose a model whose parameters fully characterize the system under investigation. The reliability of this parametric analysis depends on how well the model fits the data, which in cardiovascular applications are often assumed to be autoregressive processes $[3,4]$ or point-processes [5]. The second approach is not based on any assumption regarding the structure of the signal, and it transforms the data into a new representation that facilitates the analysis. This last approach is non-parametric.

The time-frequency analysis representations described in this chapter are non-parametric. They are used to transform non-stationary signals into joint time-frequency representations that reveal the temporal evolution of their spectral components.

Analysis of raw data through time-frequency representations can be achieved in three steps:

1) Representation of the signal or the interactions among signals in the joint time-frequency domain.

2) Localization of relevant patterns and regions of interest in this new domain.

3) Extraction of useful information, usually by transforming the relevant patterns observed in the region of interest into a time-varying physiological index or a single value.

\subsubsection{Spectral analysis and its limitations}

The spectral analysis is based on the Fourier transform, which provides a decomposition of a signal $x(t)$ into a linear combination of complex exponential functions:

$$
x(t)=\int X(f) e^{j 2 \pi t f} d f
$$

In this expression, the complex exponential functions $e^{j 2 \pi t f}$ are weighted by the complex function $X(f)$, that quantifies the spectral content of $x(t)$, i.e. the contribution of each oscillation to the signal $x(t)$. The function $X(f)$ is the Fourier transform of signal $x(t)$, and it is given by:

$$
X(f)=\int x(t) e^{-j 2 \pi f t} d t
$$

Expressions (1.1)-(1.2) operate transformations between the temporal and the spectral domains. The Fourier transform has a set of properties that makes it a widespread and versatile tool with many applications in signal processing. The most relevant are listed in Table 1.1.

The power spectral density is a representation of the distribution of the signal power over its spectral components, i.e. as a function of frequency. According to the Wiener-Khinchin theorem, the power 


\begin{tabular}{|cccc|}
\hline \hline Time & \multicolumn{4}{c|}{ Frequency } \\
$h(t)=a \cdot x(t)+b \cdot y(t)$ & $\leftrightarrow$ & $H(f)=a \cdot X(f)+b \cdot Y(f)$ & Linearity \\
$h(t)=x\left(t-t_{0}\right)$ & $\leftrightarrow$ & $H(f)=X(f) e^{-j 2 \pi t_{0} f}$ & $t$-Shifting \\
$h(t)=e^{j 2 \pi t f_{0}} x(t)$ & $\leftrightarrow$ & $H(f)=X\left(f-f_{0}\right)$ & $f$-Shifting \\
$h(t)=x(a \cdot t)$ & $\leftrightarrow$ & $H(f)=\frac{1}{|a|} X\left(\frac{f}{a}\right)$ & $t$-Scaling \\
$x(t) \in \mathbb{R}$ & $\leftrightarrow$ & $X(-f)=X^{*}(f)$ & Conjugation \\
$h(t)=x^{(n)}(t)$ & $\leftrightarrow$ & $H(f)=(j 2 \pi f)^{n} X(f)$ & Derivative \\
$h(x)=(x * y)(t)$ & $\leftrightarrow$ & $H(f)=X(f) \cdot Y(f)$ & Product/Convolution \\
$\int|x(t)|^{2} d t$ & $\leftrightarrow$ & $\int|X(f)|^{2} d f$ & Energy conservation \\
\hline \hline
\end{tabular}

Table 1.1: Useful Fourier transform properties.

spectral density of a stationary stochastic processes $x(t)$ is the Fourier transform of its autocorrelation function:

$$
S_{\mathrm{xx}}(f)=\int r_{\mathrm{xx}}(\tau) e^{-j 2 \pi \tau f} d \tau=\int \mathrm{E}\left[x(t) x^{*}(t-\tau)\right] e^{-j 2 \pi \tau f} d \tau
$$

where $\mathrm{E}[]$ is the expectation value operator and $r_{\mathrm{xx}}(\tau)$ the autocorrelation function of $x(t)$, a function that assesses the correlation between a signal and a copy of itself as a function of the delay between them. A simple estimator of the power spectral density that can be used when only one realization of finite length is available is the periodogram, which is proportional to the squared magnitude of the Fourier transform. More advanced estimators include the Welch [6] and the multitaper [7] spectra.

The power spectral density has been widely applied to the study of cardiovascular signals, in particular to the analysis of heart rate variability $[8,9,10]$. The spectral analysis of heart rate variability allows to non-invasively assess the autonomic nervous system modulation of the supra-ventricular cardiac structures responsible of heart rate regulation [11, 12, 13]. Figure 1.1 shows the power spectral density of cardiovascular signals simultaneously recorded at rest in supine position [14]. From left to right, this figure shows the spectrum of heart rate variability, respiratory signal measured as chest movement with a thoracic belt, and systolic arterial blood pressure variability. A respiratory component is present in all the three signals in the so called high-frequency spectral band (HF), $0.15-0.40 \mathrm{~Hz}$ [10], while a low-frequency component is present in the heart rate and blood pressure variability in the low-frequency spectral band (LF), $0.04-0.15 \mathrm{~Hz}$. The analysis of these two spectral components provides information regarding the parasympathetic and sympathetic activity. The HF component of the heart rate variability is considered a marker of parasympathetic activity $[10,15]$ reflecting respiratory sinus arrhythmia. The precise nature of its underlying mechanisms are still matter of debate [16]. The LF component of the heart rate variability is related to both the sympathetic and parasympathetic activity and its origin and interpretation are still not completely understood [17]. The proportion between the LF and HF component has been proposed and is often used as a marker of the sympathovagal balance [11], but its use remain controversial $[18,19,20]$.

The main limitation of spectral analysis is that it requires a signal to be stationary and does not provide any temporal localization of events. Mathematically, the lack of localization is due to the decomposition of a signal into sinusoids and cosinusoids, i.e. $e^{j 2 \pi t f}$, that are equally spread along the entire temporal axis. Figure 1.2 shows two schematic examples of this limitation. In the first one, the frequency of a signal (on the left) is increasing during time, but this dynamic feature is lost in its spectral representation (on the right), where the effect of a time-varying instantaneous frequency results in a single widespread spectral component. In the second example, the amplitude of a sinusoid (on the left) is increasing over 


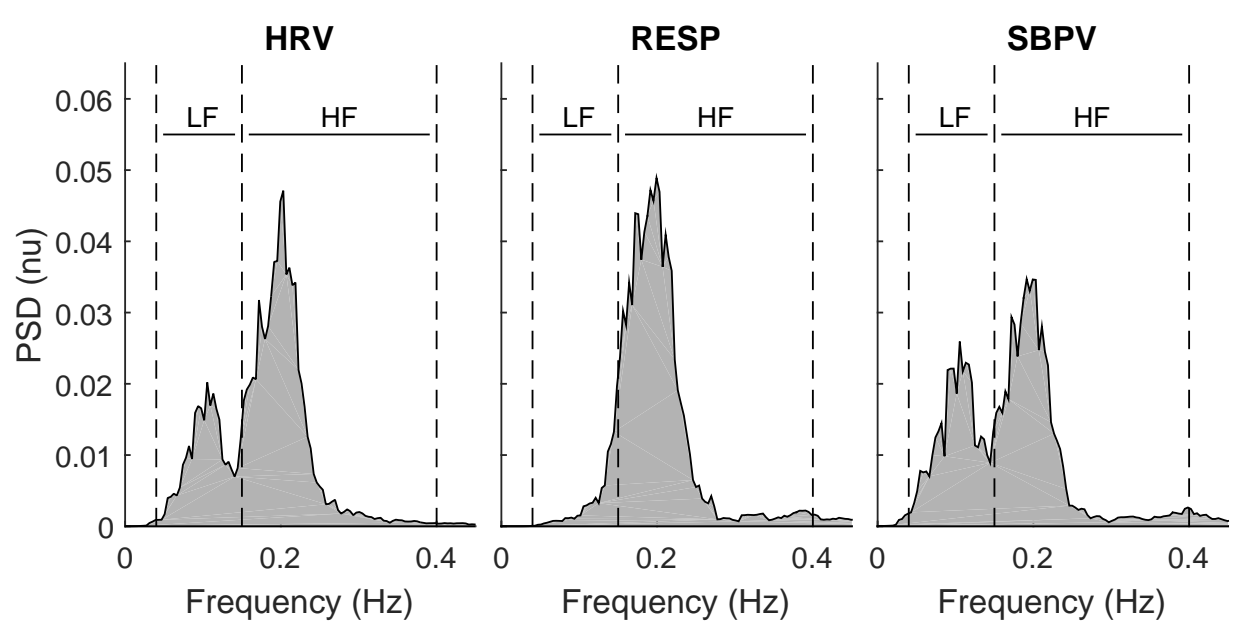

Figure 1.1: Normalized power spectral density (PSD) of : Heart rate variability (HRV), respiratory signal (RESP) measured as chest movement with a thoracic belt, and systolic arterial blood pressure variability (SBPV). A high-frequency (HF) component reflecting respiration is present in all the three spectra, while a low-frequency component (LF) is present in the HRV and SBPV. These signals have been derived from simultaneous recordings in a subject at rest in supine position. A multitaper approach has been used to estimate the PSDs.

time, but this dynamic characteristic is again lost in its spectral representation (on the right), which only shows a single static spectral peak. Another classical example of spectral analysis limitations is that the spectrum of a signal and the spectrum of the same signal recorded backward are exactly the same. The temporal organization of a signal is coded in the phase of the Fourier transform, which is not used to construct a power spectrum.

Ideally, a joint time-frequency representation should be able to track changes of the instantaneous frequencies and amplitudes of the signal's components with high time and frequency resolution. An example of an ideal time-frequency representation is reported in Figure 1.3, where a sinusoid of finite length, time-varying amplitude and frequency, and a temporal impulse are all perfectly localized in time and frequency. The instantaneous power and the power spectral density can be obtained by integrating the time-frequency representation over time (right panel) and over frequency (middle panel), respectively. These representations are called marginals. However, as it will be shown in the next section, this ideal representation is not achievable because of fundamental compromise between localization in time and frequency. 

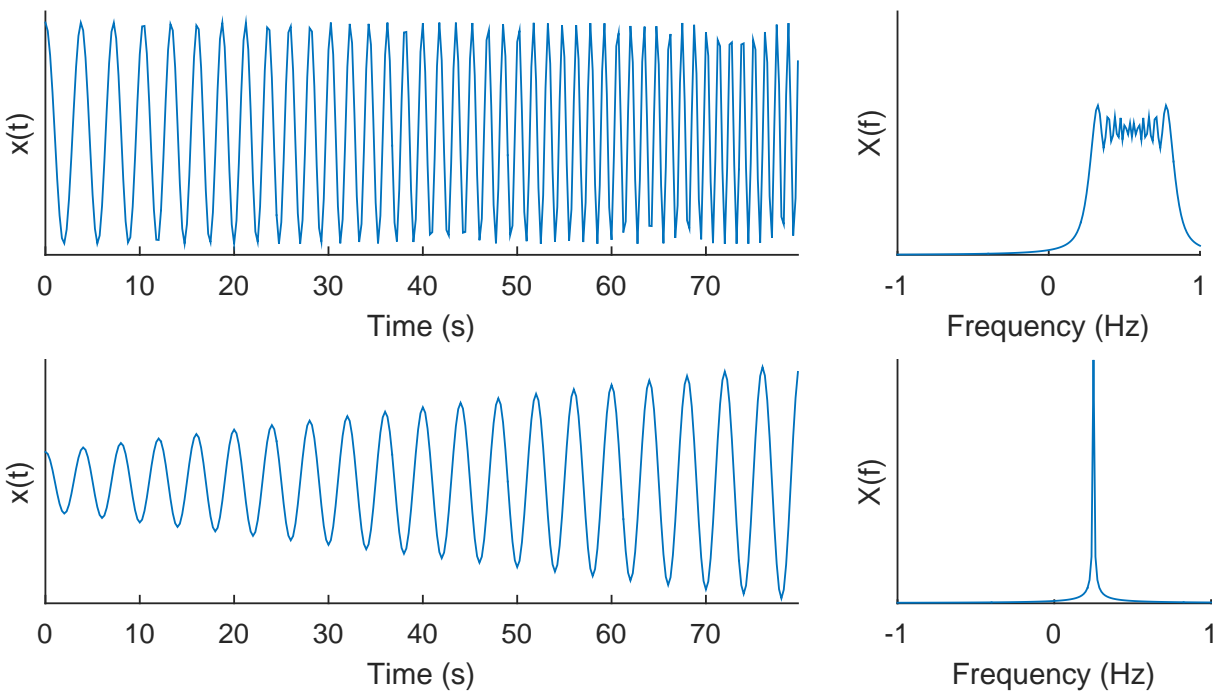

Figure 1.2: Simple examples of spectral analysis limitations. Top: A signal with increasing instantaneous frequency (on the left) and its spectrum (on the right). Bottom: A sinusoid with increasing instantaneous amplitude (on the left), and its spectrum (on the right). The dynamic properties of these two signals are not reflected in their spectral representation.
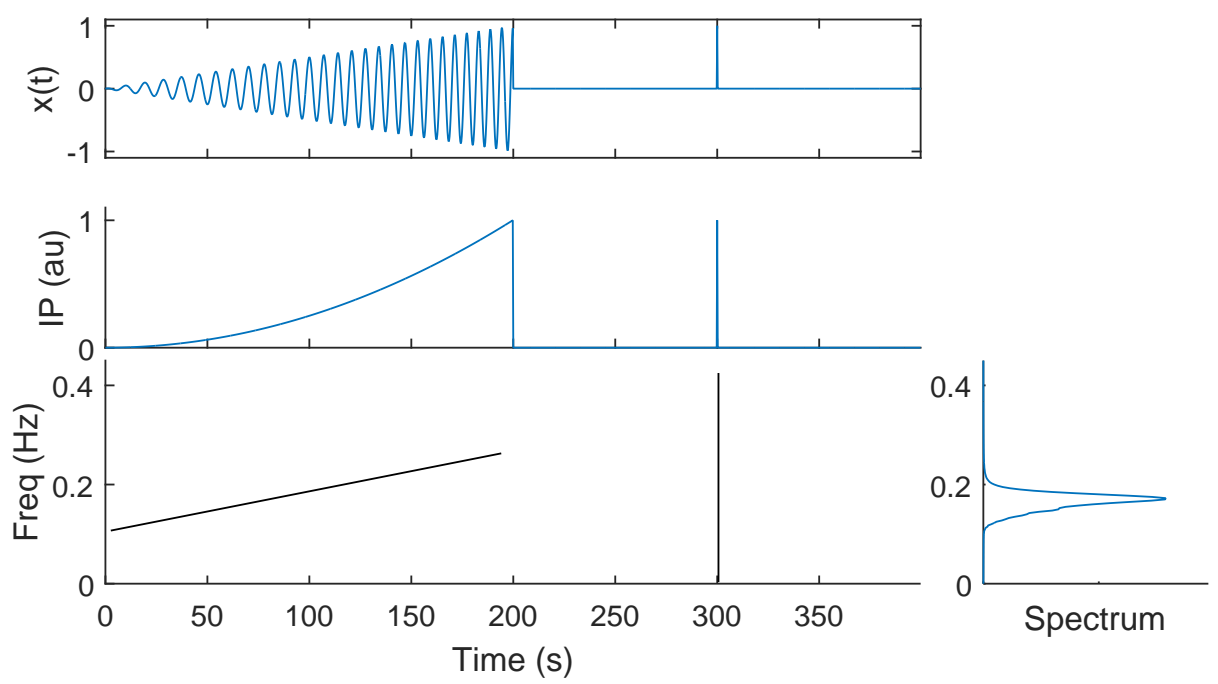

Figure 1.3: Ideal joint time-frequency representation. A signal composed of sinusoid of finite length and time-varying amplitude and frequency, plus temporal impulse is shown on the top. On the bottom, its ideal time-frequency representation reveals its time-frequency structure and provides a perfect localization of the events. The marginals obtained by integrating the time-frequency representation over time (right) and frequency (middle) represents the instantaneous power and the spectrum or the signal, respectively. 


\subsection{Studying signals: Time-frequency representations}

This section provides a detailed description of time-frequency representations belonging to the Cohen's class. A more exhaustive presentation of this subject can be found in excellent books [21, 22, 23], and reviews $[24,25]$. A useful toolbox with fundamental theory, examples and Matlab functions toolbox can be found in $[26]$.

\subsubsection{Introducing the Cohen's Class}

The time-frequency (TF) representations that belong to the Cohen's class $[24,21]$ are energy distributions, in the sense that they provide a decomposition of the signal energy (for finite energy signals), $E_{x}$, in time and frequency. A fundamental property of an energy distribution, $C_{x}(t, f)$, is that it should preserve the energy of a signal $x(t)$, such that:

$$
\iint C_{x}(t, f) d t d f=E_{x}=\int|x(t)|^{2} d t=\int|X(f)|^{2} d t
$$

Furthermore, Cohen's class distributions are energy distribution covariant by translation in time and frequency, meaning that they preserve temporal and spectral shifts, such that if a signal $x(t)$ is shifted in time and modulated in frequency, its time-frequency representation should be shifted by the corresponding amount both in time and frequency:

$$
\text { if } y(t)=x\left(t-t_{0}\right) e^{j 2 \pi f_{0}} \Rightarrow C_{y}(t, f)=C_{x}\left(t-t_{0}, f-f_{0}\right)
$$

In this expression $C_{x}(t, f)$ and $C_{y}(t, f)$ are time-frequency representations of $x(t)$ and $y(t)$, respectively. Ideally, an energy distribution should also satisfy the marginal properties, meaning that its integral over time should provide the energy spectral density of the signal, while its integral over frequency should give the instantaneous power of the signal:

$$
\begin{aligned}
& \int C_{x}(t, f) d t=|X(f)|^{2} \\
& \int C_{x}(t, f) d f=|x(t)|^{2}
\end{aligned}
$$

\subsubsection{Equivalent definitions of Cohen's class distributions}

In its general form, each time-frequency representation belonging to the Cohen's class admits at least three equivalent definitions [21]:

1) Time-delay parametrization, via $\phi_{\mathrm{t}-\mathrm{d}}(t, \tau)$ :

$$
C_{x}(t, f)=\iint \phi_{\mathrm{t}-\mathrm{d}}(t-v, \tau) x\left(v+\frac{\tau}{2}\right) x^{*}\left(v-\frac{\tau}{2}\right) e^{-j 2 \pi f \tau} d v d \tau=\int r_{x}(t, \tau) e^{-j 2 \pi f \tau} d \tau
$$

In this expression, the time-frequency distribution is seen as the Fourier transform of a nonstationary, deterministic, smoothed autocorrelation function $r_{x}(t, \tau)$ :

$$
r_{x}(t, \tau)=\int \phi_{\mathrm{t}-\mathrm{d}}(t-v, \tau) x\left(v+\frac{\tau}{2}\right) x^{*}\left(v-\frac{\tau}{2}\right) d v
$$

where $\phi_{\mathrm{t}-\mathrm{d}}(t, \tau)$ is a kernel (subscripts t-d stand for time-delay) whose function is explained in the following. Note that (1.8) is similar to the Wiener-Khinchin theorem, which demonstrates that the autocorrelation function and the power spectral density of a stochastic stationary process are Fourier transform pairs. 
2) Delay-Doppler parametrization, via $\phi_{\mathrm{d}-\mathrm{D}}(\tau, \nu)$ :

$$
C_{x}(t, f)=\iint \phi_{\mathrm{d}-\mathrm{D}}(\tau, \nu) A_{x}(\tau, \nu) e^{j 2 \pi(t \nu-f \tau)} d \nu d \tau
$$

In this expression, the time-frequency distribution is seen as the two-dimensional Fourier transform of the narrow-band symmetric Ambiguity function, $A_{x}(\tau, \nu)$, modulated by the kernel function $\phi_{\mathrm{d}-\mathrm{D}}(\tau, \nu)$ (subscripts d-D stand for delay-Doppler, the Fourier pairs of frequency and time). The narrow-band symmetric Ambiguity function is defined as [27]:

$$
A_{x}(\tau, \nu)=\int x\left(t+\frac{\tau}{2}\right) x^{*}\left(t-\frac{\tau}{2}\right) e^{-j 2 \pi \nu t} d t
$$

and it can be seen as a two variable generalization of a correlation function, with the interesting property that its magnitude is invariant to temporal and spectral shifts:

$$
\text { if } \quad y(t)=x\left(t-t_{0}\right) e^{j 2 \pi f_{0}} \Rightarrow\left|A_{y}(t, f)\right|=\left|A_{x}(t, f)\right|
$$

3) Time-frequency parametrization, via $\phi_{\mathrm{t}-\mathrm{f}}(t, f)$ :

$$
C_{x}(t, f)=\iint \phi_{\mathrm{t}-\mathrm{f}}(t-v, f-u) W_{x}(v, u) d v d u=\phi_{\mathrm{t}-\mathrm{f}}(t, f) \otimes W_{x}(t, f)
$$

In this expression, the time-frequency distribution is obtained by low-pass filtering (symbol $\otimes$ stands for 2D convolution) in time and frequency the Wigner-Ville distribution (WVD), defined as the Fourier transform of the non-stationary, deterministic, autocorrelation function:

$$
W_{x}(t, f)=\int x\left(t+\frac{\tau}{2}\right) x^{*}\left(t-\frac{\tau}{2}\right) e^{-j 2 \pi f \tau} d \tau
$$

Expression (1.13) shows that the WVD can be considered as the central element of the Cohen's class, i.e. the distribution from which all the other ones can be derived through a simple convolution with a time-frequency kernel function $\phi_{\mathrm{t}-\mathrm{f}}(t, f)$. The WVD (1.14) and the narrowband symmetric ambiguity function (1.11) are related by two dimensional Fourier transform [28]:

$$
A_{x}(\tau, \nu)=\iint W_{x}(t, f) e^{-j 2 \pi(\nu t-f \tau)} d t d f
$$

This implies that the WVD is itself a member of the Cohen's class, characterized by $\phi_{\mathrm{d}-\mathrm{D}}(\tau, \nu)=1$ and consequently $\phi_{\mathrm{t}-\mathrm{f}}(t, f)=\delta(t, f)$, i.e. a Dirac delta distribution centered at $t=0$ and $f=0$.

Every representation belonging to the Cohen's class is perfectly characterized by one of the three kernels previously introduced, i.e. $\phi_{\mathrm{t}-\mathrm{d}}(t, \tau), \phi_{\mathrm{d}-\mathrm{D}}(\tau, \nu)$ or $\phi_{\mathrm{t}-\mathrm{f}}(t, f)$. These kernels completely define the properties of the TF distributions, their time-frequency resolution, and are mutually related by the Fourier transform $[28]$ :

$$
\begin{aligned}
\phi_{\mathrm{d}-\mathrm{D}}(\tau, \nu) & =\int \phi_{\mathrm{t}-\mathrm{d}}(t, \tau) e^{-j 2 \pi \nu t} d t=\iint \phi_{\mathrm{t}-\mathrm{f}}(t, f) e^{j 2 \pi(\tau f-\nu t)} d t d f \\
\phi_{\mathrm{t}-\mathrm{f}}(t, f) & =\int \phi_{\mathrm{t}-\mathrm{d}}(t, \tau) e^{-j 2 \pi f \tau} d \tau=\iint \phi_{\mathrm{d}-\mathrm{D}}(\tau, \nu) e^{j 2 \pi(t \nu-\tau f)} d \tau d \nu
\end{aligned}
$$

The main role of these kernels is to reduce the interference terms that affect the WVD and they have been extensively studied since the early Nineties [29, 30, 31, 32, 33, 34, 35, 36].

Of note, from the WVD, by convolution with an appropriate kernel it is possible to derive:

a) Any time-frequency distribution: $C_{x}(t, f)=\phi_{\mathrm{t}-\mathrm{f}}(t, f) \otimes W_{x}(t, f)$. This implies that the geometrical properties of $\phi_{\mathrm{t}-\mathrm{f}}(t, f)$ have a major effect on the representation $C_{x}(t, f)$.

b) The spectrogram: $C_{x}(t, f)=W_{h}(t, f) \otimes W_{x}(t, f)=\left|\int x(s) h^{*}(s-t) e^{-j 2 \pi f s} d s\right|^{2}$, where $W_{h}(t, f)$ is the WVD of the sliding window $h(t)$.

c) The scalogram: $C_{x}(t, f)=W_{\psi}(t, f) \otimes W_{x}(t, f)$, where $W_{\psi}(t, f)$ is the WVD of the mother wavelet. 


\subsubsection{The Wigner-Ville Distribution and the interference terms}

The WVD defined in (1.14) is the Fourier transform of the non-stationary autocorrelation function and is the central element of the any time-frequency representation belonging to the Cohen's class. It has many interesting properties $[26,21]$, among which the most relevant are:

- Energy conservation: see (1.4).

- Satisfaction of marginal properties, see (1.6), meaning that its integration over time and frequency provides the power spectral density and the instantaneous power of the signal, respectively.

- Translation covariance, see (1.5), meaning that if a signal is shifted in time and modulated in frequency, its WVD will be shifted in time and frequency accordingly.

- Real valued: $W_{x}(t, f) \in \mathbb{R} \forall(t, f)$.

- Wide-sense support conservation:

if $x(t)=0$ for $t<|T| \Rightarrow W_{x}(t, f)=0$ for $t<|T|$;

if $X(f)=0$ for $f<|\Omega| \Rightarrow W_{x}(t, f)=0$ for $f<|\Omega|$.

- Perfect localization of linear chirps, i.e. a complex exponential whose instantaneous frequency $\theta(t)$ is a linear function of time :

if $x(t)=e^{j 2 \pi \theta(t) t}, \theta(t)=a+\beta / 2 t \Rightarrow W_{x}(t, f)=\delta(f-(a+\beta t))$

However, its use is seriously limited by the presence of interference terms. Interferences are oscillatory terms inherent to the quadratic structure of the WVD, which do not represent signal spectral components but are due to their mutual interactions [37, 38]. Therefore, they are usually considered as artifacts that should be eliminated, or at least reduced, in order to improve the readability of the distribution. Interference terms are a consequence of the WVD's bilinear (or quadratic) structure and they can be identified mathematically with quadratic cross-terms [39, 37]. They appear whenever a signal can be described as the sum of different spectral components or distinct spectral atoms. For example, the WVD of a signal $x(t)=x_{1}(t)+x_{2}(t)$, is:

$$
W_{x}(t, f)=W_{1}(t, f)+W_{2}(t, f)+2 \Re\left[W_{12}(t, f)\right]
$$

where $W_{1}(t, f)$ and $W_{2}(t, f)$ are the WVDs of $x_{1}(t)$ and $x_{2}(t)$, also called auto-terms because they represent signal components, whereas $W_{12}(t, f)$ is an interference term that oscillates with a frequency and in a direction that depend on the relative position of the auto-terms in the TF plane $[37,38,25]$. The geometry of the interference terms can be explained considering two TF atoms $x_{1}(t)=h(t-$ $\left.t_{1}\right) e^{j 2 \pi f_{1} t}$ and $x_{2}(t)=h\left(t-t_{2}\right) e^{j 2 \pi f_{2} t}$, where $h(t)$ is a Gaussian window, well localized around $\left(t_{1}, f_{1}\right)$ and $\left(t_{2}, f_{2}\right)$. The WVD of their sum $x(t)=x_{1}(t)+x_{2}(t)$ includes an interference term with the following characteristics:

- It is centered around $\left(t_{12}, f_{12}\right)=\left(\frac{t_{2}+t_{1}}{2}, \frac{f_{2}+f_{1}}{2}\right)$.

- It oscillates in a direction perpendicular to the axis connecting $\left(t_{1}, f_{1}\right)$ and $\left(t_{2}, f_{2}\right)$.

- Its frequency in time direction is proportional to $\left|f_{2}-f_{1}\right|$.

- Its frequency in frequency direction is proportional to $\left|t_{2}-t_{1}\right|$.

Therefore, the spectral and temporal distances between the TF atoms, $\left|f_{2}-f_{1}\right|$ and $\left|t_{2}-t_{1}\right|$, determine the the velocity of the oscillation of the interference term.

Interference terms are conceptually divided in two groups: outer and inner interferences [38]. Outer interferences appear in multi-component signals, as the result of the interactions between spectral components that are present at the same time. They are usually located at midway between two components and 
they mainly oscillate in time direction. Inner interferences are due to non-linearities in the instantaneous frequency of a single spectral component. Their structure obeys the same geometric laws of outer interferences. Figure 1.4 provides a graphical explanation of the geometry of the interference terms using two examples. Both panels show the WVD of a signal composed of two TF atoms $x_{1}(t)=h\left(t-t_{1}\right) e^{j 2 \pi f_{1} t}$ and $x_{2}(t)=h\left(t-t_{2}\right) e^{j 2 \pi f_{2} t}$, with $t_{1}<t_{2}$ and $f_{1}<f_{2}$ and where $h(t)$ is a Gaussian window. In both cases the WVD provides a good localization of atoms $x_{1}(t)$ and $x_{2}(t)$, but it is affected by interference terms. The geometrical center of the interference terms is in the middle of the auto-terms at $\left[\left(t_{1}+t_{2}\right) / 2,\left(f_{1}+f_{2}\right) / 2\right]$ and the oscillation propagates along a direction perpendicular to the axis connecting the auto-terms. The interference oscillations in both time and frequency directions are faster in the example shown in the upper panel than in the example shown in the lower one because both $\left|t_{2}-t_{1}\right|$ and $\left|f_{2}-f_{1}\right|$ are larger in the latter. This is better represented in the panels on the right-hand side, which show slices of the WVD evaluated at time and frequency that correspond to the centers of the auto- and cross-terms. Here again it is clear that the velocity of the interference oscillation increases with the distance between the auto-terms.

The oscillatory nature of the interference terms suggests that they can be selectively attenuated or eliminated by filtering the WVD with kernels that act as low-pass filters. The interpretation of the kernels in (1.16)-(1.17) as low-pass filters imposes geometrical constraints on their design, and algorithms that use specific signal properties to automatically determine an optimal kernel, so called signal-dependent kernels, have been proposed $[35,30,31,33,32,40]$. One strategy is to determine the properties of the low-pass filter based on the minimum time-frequency distance between the signal components, which is related to the lowest oscillation to be eliminated. For example, energy time-frequency distributions of cardiovascular signals are expected to present both outer and inner interferences. Outer interferences arise from the interactions between simultaneous LF and HF oscillations, while inner interferences may arise from non-linearities in the instantaneous frequencies of this components. Outer interferences may be eliminated by estimating the minimum distance between $\left|f_{\mathrm{HF}}(t)-f_{\mathrm{LF}}(t)\right|$, which is usually determined by the respiratory rate, and then by using it to determine the cut-off of the low-pass filter in the time direction [40].

Finally, it is worth mentioning that the complex analytic representation of the signal is often used instead of the original real signal to further reduce the interference terms [41]. This is because the power spectral density of the analytic representation of a signal vanishes for $f<0$. Therefore, the use of the complex analytic signal assures that positive and negative spectral components do not produce interference terms and improves the readability of the TF distribution.

\subsubsection{Time-frequency resolution}

If we consider the general scheme for the analysis of non-stationary signals shown in Sec. 1.1, it is clear that the final result of the analysis is strictly related to the time-frequency resolution of the representation. For example, in the analysis of cardiovascular signals, the frequency resolution should be good enough to separate the LF and the HF components, even when low respiratory rate reduces their spectral distance. At the same time, time resolution should be good enough to follow quick variations, typically of the order of few seconds.

The time-frequency resolution of a distribution belonging to the Cohen's class depends on the degree of filtering provided by the kernel function, which, in turn, depends on its geometrical properties.

Although in theory the use of bespoke kernels offers the possibility to go beyond the Heisenberg principle, which states that time and frequency resolutions cannot be jointly arbitrarily low because they are inversely related, the violation of this principle introduces cross-terms. Thus, the classical trade-off between time and frequency resolution becomes a trade-off between joint time-frequency resolution and 

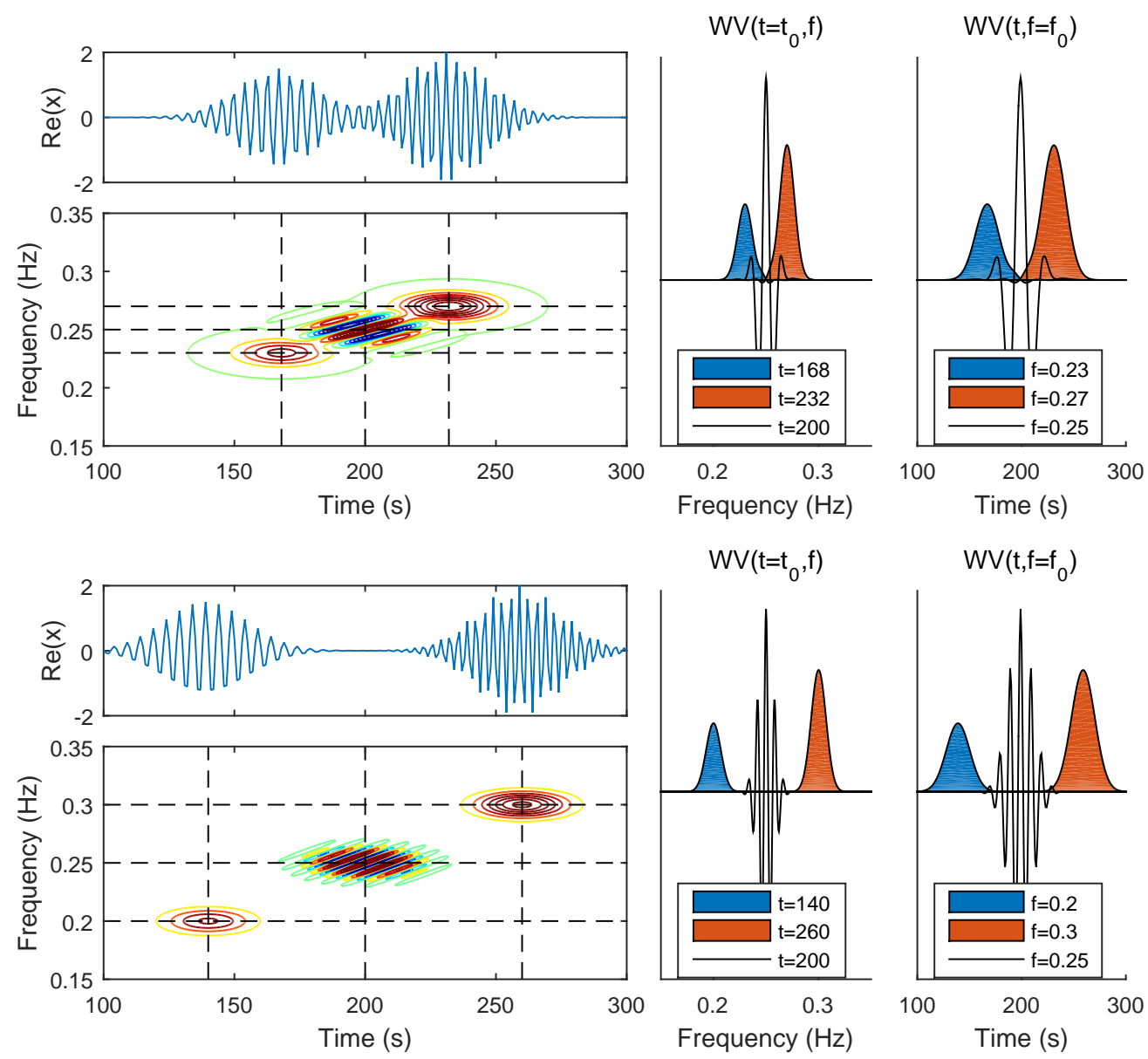

Figure 1.4: Graphical explanation of the geometry of the interference terms. On the left: A signal composed of two time-frequency atoms $x_{1}(t)$ and $x_{2}(t)$, with $t_{1}<t_{2}$ and $f_{1}<f_{2}$, and where $h(t)$ is a Gaussian window, and its Wigner-Ville distribution. On the right: The Wigner-Ville distribution evaluated at time and frequency corresponding to the geometrical centers of auto- and interference terms. The WVD provides a good localization of atoms $x_{1}(t)$ and $x_{2}(t)$, but it is affected by interference terms. The geometrical center of the interference terms is in the middle of the auto-terms and the oscillation propagates along a direction perpendicular to the axis connecting the auto-terms. The interference oscillations in both time and frequency direction are faster in the example in the upper than in the bottom panel because the temporal and spectral distance between the atoms are larger in the latter. This is better represented in the panels on the right-hand side, which show slices of the WVD evaluated at time and frequency that correspond to the centers of the auto- and cross-terms. Here again it is clear that the velocity of the interference oscillation increases with the distance between the auto-terms. 
amount of cross-terms. As we will see in the following, this is paticularly relevant when time-frequency distributions are used to estimate time-frequency spectra, mainly because positivity, a necessary condition for a distribution to be interpreted as an energy spectral density, requires an appropriate amount of filtering both in time and frequency [42]. Furthermore, the amount of smoothing also depends on the signals, being higher for those signals which present more interference terms [38, 43].

The quantification of the resolution is important because it allows to compare the performance of different distributions and to chose the most appropriate for a given application, and, importantly, it is necessary for a correct interpretation of the results. For example, to correctly assess the latency between a stimulus and a response in an index extracted from a time-frequency distribution it is necessary to know the effect of the smoothing kernel on the localization of the observed features.

Since a time-frequency distribution can be obtained by the convolution between the WVD of a signal and a kernel $\phi_{\mathrm{t}-\mathrm{f}}(t, f)$ (see (1.13)), and because the WVD is a non-filtered representation, the resolution of a time-frequency distribution can be calculated by studying $\phi_{\mathrm{t}-\mathrm{f}}(t, f)$. The full-width at half maximum or any other measurement of the spreading of the kernel in time and frequency provides an estimate of the time-frequency resolution [14]. This can be easily seen by considering the example of the time-frequency distribution of a temporal impulse $\delta\left(t-t_{0}\right)$ or of a complex exponential $e^{j 2 \pi f_{0} t}$. The WVD of these two signals is a line along frequency and time, respectively. The convolution with $\phi_{\mathrm{t}-\mathrm{f}}(t, f)$ results in a time-frequency representation which is the same $\phi_{\mathrm{t}-\mathrm{f}}(t, f)$ centered around $t_{0}$ or $f_{0}$, respectively.

\subsubsection{The smoothed-pseudo Wigner-Ville distribution (SPWVD)}

Several filtered versions of the WVD have been proposed to reduce the interference terms while maintaining a good TF resolution. Among those that have been applied to the study of the cardiovascular variability $[44,45]$, the smoothed pseudo Wigner-Ville distribution (SPWVD) is one of the most interesting, since it provides an independent control over time and frequency resolution. The SPWVD is the only member of the Cohen's class that makes use of separable kernels, i.e. $\phi_{\mathrm{t}-\mathrm{f}}(t, f)=\phi_{\mathrm{t}}(t) \phi_{\mathrm{f}}(f)$, and offers the possibility to independently control the amount of time and frequency filtering [41, 21, 24]. The SPWVD of a signal $x(t)$ can be estimated in the time-lag domain $(t, \tau)$ by means of $(1.8)$ and by using the separable kernel $\phi_{\mathrm{t}-\mathrm{d}}(t, \tau)=\phi_{\mathrm{t}}(t) \phi_{\mathrm{d}}(\tau)$ :

$$
C_{x}(t, f)=\int \phi_{\mathrm{d}}(\tau)\left[\int \phi_{\mathrm{t}}(t-v) x\left(v+\frac{\tau}{2}\right) x^{*}\left(v-\frac{\tau}{2}\right) d v\right] e^{-j 2 \pi f \tau} d \tau
$$

In this expression, the term in the brackets represents a smoothed version of the non-stationary deterministic autocorrelation function. The time and the frequency resolution of the SPWVD is separately determined by the shape of $\phi_{\mathrm{t}}(t)$ and by the shape of the Fourier transform of $\phi_{\mathrm{d}}(\tau)$, respectively.

Figure 1.5 provides an illustrative example of the effect of using different smoothing strategies. Panels on the upper line represent a signal composed of 4 Gaussian atoms, i.e. 4 complex exponentials modulated by Gaussian functions. Graphics (A)-(D) show 4 different TF distributions of this signal. The centers of the TF atoms are localized by black crosses. Panel (A) shows the WVD. The 4 TF atoms are well localized both in time and frequency. However, several interference terms also appear in between the atoms. Importantly, some of these interference terms have an amplitude higher than the amplitude of the auto-terms related to the TF atoms. In panel (B) and (C) the WVD is only filtered in frequency and time, respectively. It is shown that the filtering in frequency cancels the interference terms arising from the interaction of TF atoms with the same central frequencies (see panel (B)), while the filtering in the time direction cancels the interferences terms arising from the interactions of TF atoms with the same central time (see panel $(\mathrm{C})$ ). If we compare the distributions in $(\mathrm{B})-(\mathrm{C})$ with the WVD in $(\mathrm{A})$, we observe that the filtering reduces the localization in frequency and time, respectively. Finally, in panel 


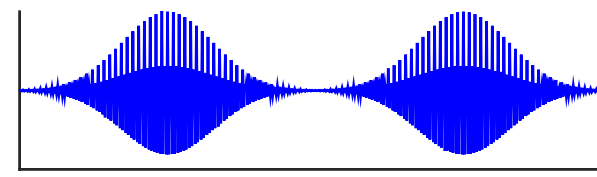

(A) WVD

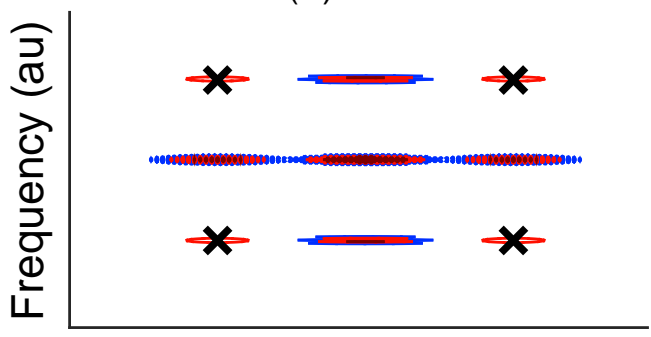

(C) SWVD
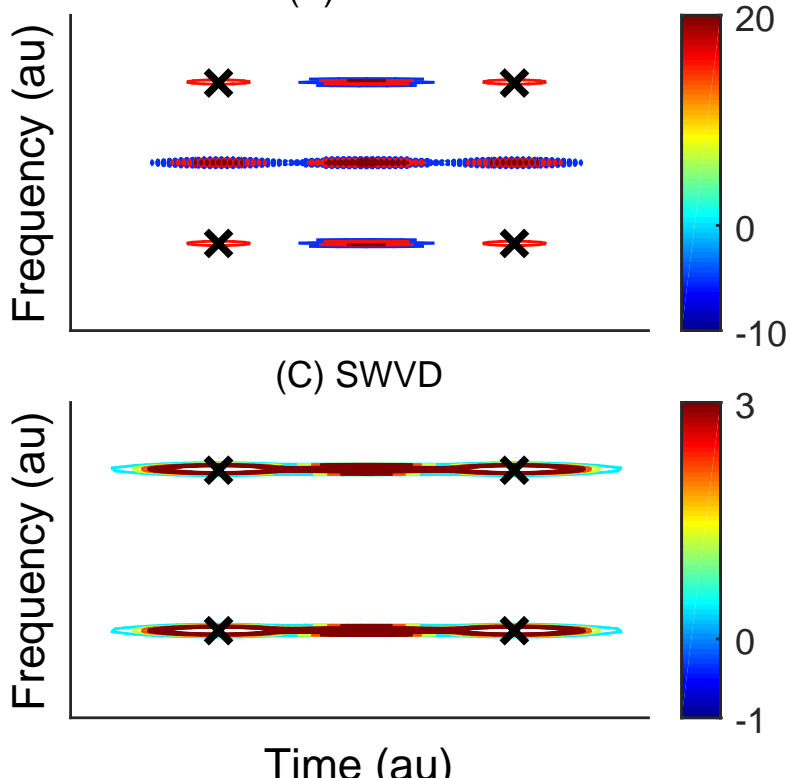

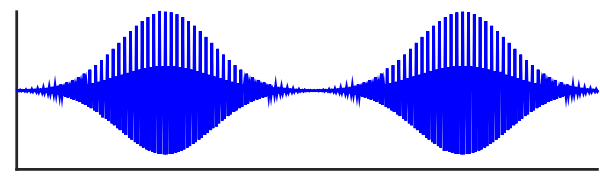

(B) PWVD

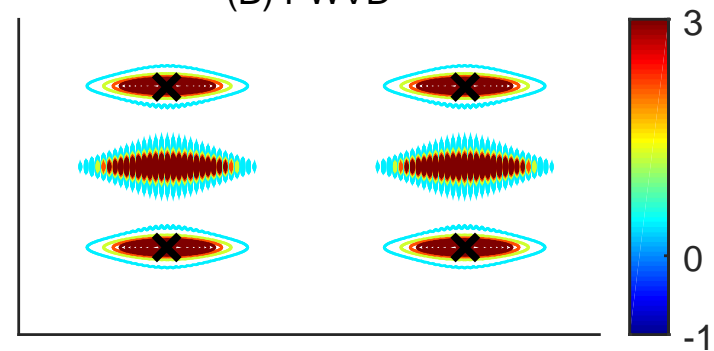

(E) SPWVD

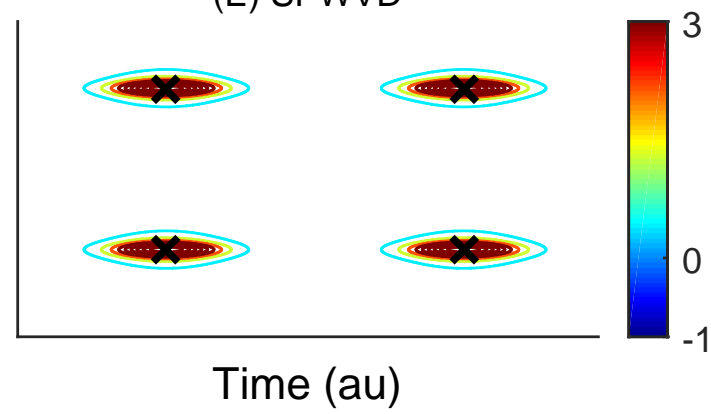

Figure 1.5: Effect of time-frequency smoothing. Upper line: All panels represent a signal composed of 4 Gaussian atoms. (A)-(D): Iso-contours of time-frequency representation with different degree of filtering. Black cross localize the center of the Gaussian atoms in the time-frequency domain. Timefrequency distributions are normalized to the total energy of the signal. (A): Wigner-Ville distribution (no smoothing). (B): Pseudo Wigner-Ville distribution (frequency smoothing only). Note that interference terms oscillating in the frequency direction and centered between the horizontal lines connecting the atoms have been eliminated. (C): Smoothed Wigner-Ville distribution (time smoothing only). Note that interference terms oscillating in the time direction and centered between the vertical line connecting the atoms have been eliminated. (D): Smoothed pseudo Wigner-Ville distribution (both time and frequency smoothing). All interference terms have been removed at a cost of a increased smoothing. 
(D), the SPWVD obtained by combining the time and frequency filtering used in (B) and (C) is shown. In this representation, all the interference terms have been eliminated. Additionally, the smoothing applied to the distribution shown in (D) was sufficient to make the SPWVD positive, so that it can now be considered as an estimate of the non-stationary spectrum of signal $x(t)$.

\subsubsection{From time-frequency representations to time-frequency spectra}

The definition of the power spectral density of a stationary random process is given in (1.3). Contrary to the stationary case, a non-stationary spectrum for a time-varying random process is not uniquely defined. The Wigner-Ville spectrum (WVS), defined as the Fourier transform of a non-stationary auto-correlation function [21]:

$$
S_{\mathrm{xx}}(t, f)=\int \mathrm{E}\left[x\left(t+\frac{\tau}{2}\right) x^{*}\left(t-\frac{\tau}{2}\right)\right] e^{-j 2 \pi \tau f} d \tau
$$

can be seen as a natural extension of the power spectral density in a non-stationary context. It can be shown that, under mild conditions, the Wigner-Ville spectrum (1.20) is equal to the ensemble average of the Wigner-Ville distributions (WVD), $W_{\text {xx }}(t, f)$ (see (1.14)), of the realizations of the processes [21]:

$$
S_{\mathrm{xx}}(t, f)=\mathrm{E}\left[W_{\mathrm{xx}}(t, f)\right]
$$

When only one observed realization is available, $S_{\mathrm{xx}}(t, f)$ should be estimated by replacing the expectation $\mathrm{E}\left[W_{\mathrm{xx}}(t, f)\right]$ with local averaging. This can be achieved by using Cohen's class distributions:

$$
\hat{S}_{\mathrm{xx}}(t, f ; \phi)=W_{\mathrm{xx}}(t, f) \otimes \phi_{\mathrm{t}-\mathrm{f}}(t, f)
$$

where $\otimes$ represents the $2 \mathrm{D}$ convolution on $t$ and $f$, and $\phi_{\mathrm{t}-\mathrm{f}}(t, f)$ is the smoothing function, which should guarantee the positiveness of the estimated spectra. 


\subsection{Studying interactions: Multivariate time-frequency repre- sentations}

This section describes time-frequency methodologies utilized to study the dynamic interactions between non-stationary signals. These include:

1) Time-frequency coherence: For the analysis of the strength of the local coupling between two signals.

2) Time-frequency phase-difference: For the analysis of the degree of synchronization between two signals.

3) Time-frequency phase-locking: For assessing the degree of coordination between multiple responses.

4) Time-frequency partial spectrum: For assessing the variability of a given signal after removing the amount of variability in synchrony with another signal.

Most of the methodologies presented in this section have been specifically proposed to study dynamic interactions between cardiovascular signals $[14,2,46]$.

In the following, when a time-frequency distribution represents a time-varying power spectrum, the symbol $S(t, f)$ will be used instead of $C(t, f)$.

\subsubsection{Time-frequency coherence}

Spectral coherence measures the degree of correlation between the spectral components of two signals [47]. This measure requires signals to be stationary and it is therefore inappropriate for studying nonstationary signals. In the analysis of cardiovascular signals, the estimation of spectral coherence in the joint time-frequency (TF) domain has many potential fields of application. For example, the localization of $\mathrm{TF}$ regions in which two signals are coupled can be applied in the time-varying characterization of baroreflex $[48,49,50]$, or in the assessment of the degree of similarity between different signals to validate the use of one signal, and its derived measures, as surrogates of original ones [51].

The spectral coherence function between two stationary zero-mean stochastic processes $x(t)$ and $y(t)$ is a normalized version of the cross power spectral density, $S_{\mathrm{xy}}(f)$, which measured the cross-correlation between two different signals as a function of frequency and is estimated as in (1.3). The spectral coherence is defined as:

$$
\gamma(f)=\frac{S_{\mathrm{xy}}(f)}{\sqrt{S_{\mathrm{xx}}(f) S_{\mathrm{yy}}(f)}},
$$

The magnitude of the spectral coherence is equal to one for those frequencies for which $x(t)$ and $y(t)$ are related by a linear time-invariant system and it is equal to zero for those frequencies for which $x(t)$ and $y(t)$ are uncorrelated, i.e. when $S_{\mathrm{xy}}(f)=0[47,52]$.

By analogy with the stationary case, the time-frequency coherence (TFC) can be defined in nonstationary conditions as [53, 52]:

$$
\gamma(t, f)=\frac{S_{\mathrm{xy}}(t, f)}{\sqrt{S_{\mathrm{xx}}(t, f) S_{\mathrm{yy}}(t, f)}}
$$

where $S_{\mathrm{xy}}(t, f)$ is the non-stationary cross spectrum, and $S_{\mathrm{xx}}(t, f)$ and $S_{\mathrm{yy}}(t, f)$ are the non-stationary auto-spectra estimated as in (1.22). A critical aspect of the estimation of the TFC is the elimination of the interference terms, as these may cause the TFC to take values outside the range $[0,1]$, thus losing its physical meaning. This is shown by the Janssen's formula (also known as outer interference formula) $[38,21]$ :

$$
\left|W_{\mathrm{xy}}(t, f)\right|^{2}=\iint W_{\mathrm{xx}}\left(t+\frac{\tau}{2}, f+\frac{\nu}{2}\right) W_{\mathrm{yy}}\left(t-\frac{\tau}{2}, f-\frac{\nu}{2}\right) d \tau d \nu
$$


This expression shows that for given $\mathrm{TF}$ point $\left(t_{0}, f_{0}\right)$, it is possible to have $\left|W_{\mathrm{xy}}\left(t_{0}, f_{0}\right)\right| \neq 0$ while $W_{\mathrm{xx}}\left(t_{0}, f_{0}\right)=W_{\mathrm{yy}}\left(t_{0}, f_{0}\right) \approx 0$, and consequently $\gamma\left(t_{0}, f_{0}\right)>1$.

To obtain TFC estimates bounded between zero and one, $\phi_{\mathrm{t}-\mathrm{f}}(t, f)$ should completely suppress the interference terms [52]. As long as the degree of TF filtering is strong enough, TF coherence can be obtained using (1.24).

In the analysis of the interactions between cardiovascular signals, a simplified version of the multiformtiltable exponential kernel first proposed in [33], was succesfully used [40, 51, 14, 2, 46]:

$$
\phi_{\mathrm{d}-\mathrm{D}}(\tau, \nu)=\exp \left\{-\pi\left[\left(\frac{\nu}{\nu_{0}}\right)^{2}+\left(\frac{\tau}{\tau_{0}}\right)^{2}\right]^{2 \lambda}\right\}
$$

In the ambiguity function domain, the iso-contours of this kernels are ellipses whose eccentricity depends on parameters $\nu_{0}$ and $\tau_{0}$. Parameters $\nu_{0}$ and $\tau_{0}$ are used to change the length of the ellipse axes aligned along $\nu$ (i.e. the degree of time filtering) and $\tau$ (i.e. the degree of frequency filtering), respectively. The parameter $\lambda$ sets the roll off of the filter as well as the size of the tails of the kernel.

A signal-dependent kernel that provides a time-frequency coherence bounded between 0 and 1 can be empirically determined as follows. First, the target TF resolution corresponding to the minimum amount of TF smoothing is decided based on a-priori information about the signals and the experimental settings. The set of parameters $\left\{\tau_{0}, \nu_{0}, \lambda\right\}$ associated with this TF resolution are used as a starting point. If using this set of parameters $\gamma(t, f) \notin[0,1]$, the degree of time (or frequency) smoothing is increased until reaching the minimum degree of smoothing associated with meaningful TFC estimates.

Despite the fact that the degree of time and frequency filtering can be tuned almost independently, the resulting time and frequency resolution can not be set arbitrarily, since the condition of having $\gamma(t, f) \in[0,1]$ imposes a trade of on the minimum joint TF filtering.

A more sophisticated signal-dependent scheme to automatically find a kernel associated to TFC functions bounded between 0 and 1 was proposed in [40].

\section{Statistical assessment of coherence significance}

As for the classical spectral coherence, time-frequency coherence estimates are not meaningful per se, but they strongly depend on the parameters used in its calculation $[47,54]$. The local averaging provided by the smoothing function $\phi_{\mathrm{t}-\mathrm{f}}(t, f)$ introduces a bias in the TFC estimates, causing the TFC of two uncorrelated signals to be higher than zero. This dependence introduces an uncertainty in the interpretation of the coherence level. To reduce this uncertainty and to localize TF regions characterized by a significant coherence level, a hypothesis test can be used. The test is based on the point-by-point comparison of the time-frequency coherence estimates, $\gamma(t, f)$, with a threshold function, $\gamma_{\mathrm{TH}}(t, f)$, obtained by estimating the time-frequency coherence between several realizations of locally uncoupled signals. The null hypothesis $H_{0}$ to be rejected is that two signals $x(t)$ and $y(t)$, with time-frequency coherence $\gamma(t, f)$, are uncorrelated around a point $\left(t_{0}, f_{0}\right)$. The procedure is based on surrogate data analysis, where a surrogate is a signal that shares similar features as the original one but lack of the property that needs to be tested $[55,54]$. In this case, the analysis consists of the following steps [14]:

1) Generate pairs of uncorrelated test signals $\left\{\tilde{x}_{\mathrm{j}}(t), \tilde{y}_{\mathrm{j}}(t)\right\}$.

2) Compute the distribution $\Gamma(t, f)=\left\{\gamma_{1}(t, f), \ldots, \gamma_{\mathrm{j}}(t, f), \ldots\right\}$, where $\gamma_{\mathrm{j}}(t, f)$ is the TFC between the $j$-th realization of test signals $\tilde{x}_{\mathrm{j}}(t)$ and $\tilde{y}_{\mathrm{j}}(t)$;

3) Estimate the threshold $\gamma_{\mathrm{TH}}(t, f ; \alpha)$, corresponding to the desired significance level $\alpha$. According to this framework, $\alpha$ is the probability of wrongly rejecting the null hypothesis, and $\gamma_{\text {тн }}(t, f ; \alpha)$ is estimated point-by-point as the $(1-\alpha)$-th percentile of $\Gamma(t, f)$; 
4) Determine the TF region $\mathcal{R}(t, f ; \alpha)$ in which the null hypothesis can be rejected as that where $\gamma(t, f)>\gamma_{\mathrm{TH}}(t, f ; \alpha)$.

When the test signals $\tilde{x}_{\mathrm{j}}(t)$ and $\tilde{y}_{\mathrm{j}}(t)$ are white noises the threshold function only depends on the kernel and it is expected to be constant over the entire time-frequency domain. If one of the test signals is replaced by one of the original signals, the threshold function will be signal-dependent and it should be estimated for each pair of signals even when the same kernel function is used for the analysis [14].

\subsubsection{Time-frequency phase differences}

Phase differences represent the delay between two oscillations that share approximately the same frequency. This delay, which should be much smaller than the oscillation period, can vary during time as a response to a given stimulus and may provide valuable information about the system under investigation. To introduce the time-frequency phase difference and its estimation, let's model two signals as AM-FM modulated complex exponentials with time-varying LF and HF components:

$$
\begin{aligned}
& x(t)=A_{x, \mathrm{LF}}(t) e^{j \theta_{\mathrm{x}, \mathrm{LF}}(t)}+A_{\mathrm{x}, \mathrm{HF}}(t) e^{j \theta_{\mathrm{x}, \mathrm{HF}}(t)} \\
& y(t)=A_{\mathrm{y}, \mathrm{LF}}(t) e^{j \theta_{\mathrm{y}, \mathrm{LF}}(t)}+A_{\mathrm{y}, \mathrm{HF}}(t) e^{j \theta_{\mathrm{y}, \mathrm{HF}}(t)}
\end{aligned}
$$

In these expressions, LF and HF indicate the low frequency component, traditionally inside $0.04-0.15$ $\mathrm{Hz}$, and the high frequency component, traditionally inside $0.15-0.4 \mathrm{~Hz}$, respectively [10]; $\theta_{\mathrm{k}, \mathrm{B}}(t)$, with $B \in[L F, H F]$ and $k \in[x, y]$, is the instantaneous phase, related to the instantaneous frequency by $f_{\mathrm{k}, \mathrm{B}}(t)=\left(d \theta_{\mathrm{k}, \mathrm{B}}(t) / d t\right) /(2 \pi)$.

The phase difference between these spectral components, $\theta_{\mathrm{B}}(t)=\theta_{\mathrm{x}, \mathrm{B}}(t)-\theta_{\mathrm{y}, \mathrm{B}}(t)$, is expected to be time-varying. The time-course of the phase difference between two signals $x(t)$ and $y(t)$, evaluated for a specific spectral component $B$, can be estimated as:

$$
\theta_{\mathrm{B}}(t)=2 \pi \int_{0}^{\mathrm{t}}\left[f_{\mathrm{x}, \mathrm{B}}(\tau)-f_{\mathrm{y}, \mathrm{B}}(\tau)\right] d \tau
$$

However, the estimation of the phase difference from the instantaneous frequencies of the spectral components of two signals is very sensitive to estimation errors in $f_{\mathrm{k}, \mathrm{B}}(t)$, since an estimation error at $t_{0}$ affects all $\theta_{\mathrm{B}}\left(t>t_{0}\right)$. The characterization of the phase differences in the joint time-frequency domain may therefore be preferable. The time-frequency representation of the phase difference between two signals is defined as:

$$
\Theta(t, f)=\arg \left[S_{\mathrm{xy}}(t, f)\right]=\arctan \left[\frac{\Im\left[S_{\mathrm{xy}}(t, f)\right]}{\Re\left[S_{\mathrm{xy}}(t, f)\right]}\right]
$$

This expression provides a quantification of the phase difference between the spectral components of two signals in the time-frequency domain and is bounded $\Theta(t, f) \in[-\pi, \pi]$.

Other approaches to estimate the phase differences between 2 non-stationary signals have used the wavelet transform [56, 57, 58,59], the Rihaczek transform [60,61] and the reduced interference distributions $[62,29]$.

A time-varying index describing the temporal evolution of the phase difference between a given spectral component $B$ of two signals can be estimated by averaging the time-frequency representation of the phase difference in a specific time-frequency region $\Omega_{\mathrm{B}}^{(\theta)}$. Among a wide range of different possibilities, one that has been previously used is $\Omega_{\mathrm{B}}^{(\theta)}$ defined as a time-frequency region centered around the instantaneous frequency of a spectral component of the cross spectrum, $f_{\mathrm{B}}^{(\mathrm{xy})}(t)$ (estimated as the maximum of the instantaneous spectral peak) in which coherence is statistically significant [2]. These conditions are necessary to obtain robust and accurate estimates since the estimation of a phase difference is relevant 
only in those time intervals in which the two signals are locally coupled and therefore are sharing approximately the same instantaneous frequency. This region can be defined as [2]:

$$
\Omega_{\mathrm{B}}^{(\theta)} \equiv\left\{(t, f) \mid f=f_{\mathrm{B}}^{(\mathrm{xy})}(t) \pm \frac{\Delta_{\mathrm{f}}}{2} \cap \gamma(t, f)>\gamma_{\mathrm{TH}}(t, f)\right\} ;
$$

where $\Delta_{\mathrm{f}}$ is the width of the spectral band centered around $f_{\mathrm{B}}^{(\mathrm{xy})}(t)$, which should be related to the frequency resolution of the TF distributions. The index that quantifies the phase difference, $\theta_{\mathrm{B}}(t)$, can be estimated (in radians) by averaging the time-frequency phase difference spectrum in $\Omega_{\mathrm{B}}^{(\theta)}$ :

$$
\theta_{\mathrm{B}}(t)=\left[\int_{\Omega_{\mathrm{B}}^{(\theta)}} \Theta(t, f) d f\right] /\left[\int_{\Omega_{\mathrm{B}}^{(\theta)}} d f\right] ;
$$

The time delay associated to $\theta_{\mathrm{B}}(t)$ can be estimated (in seconds) by the index $\mathcal{D}_{\mathrm{B}}(t)$, defined as:

$$
\mathcal{D}_{\mathrm{B}}(t)=\frac{\theta_{\mathrm{B}}(t)}{2 \pi f_{\mathrm{B}}^{(\mathrm{xy})}(t)}
$$

Assessing TF coherence and phase differences: In the following, the results of a simulation study designed to assess TF coherence and phase difference estimators is proposed. The purpose of the study is to demonstrate the potential of these methodologies to track fast changes in the dynamic interactions between cardiovascular signals.

The signals used in the simulation are modified versions of the RR variability signals recorded during the tilt table test [2]. They are obtained as:

$$
\begin{aligned}
& x(t)=a_{\mathrm{RRV}}(t)+\xi_{\mathrm{x}}(t) \\
& y(t)=\left[\gamma_{0}(t) \alpha_{0}(t) \exp \left(j \theta_{0}(t)\right)\right] a_{\mathrm{RRV}}(t)+\xi_{\mathrm{y}}(t)
\end{aligned}
$$

where $a_{\mathrm{RRV}}(t)$ is the complex analytic signal representation of the RRV signal, and $\xi_{\mathrm{x}}(t)$ and $\xi_{\mathrm{y}}(t)$ are complex white Gaussian noises whose amplitude is set to give a $S N R=10 \mathrm{~dB}$. Indices $\gamma_{0}(t) \in\{0,1\}$ and $\theta_{0}(t) \in[-2.2,-0.8]$ rad represent the reference time-course of the local coupling and phase difference, respectively. Index $\alpha_{0}(t)$ is a time-varying amplitude modulation. The estimation of these indices is challenging because: the TF structure of the signals reflects the complexity of real non-stationary biomedical signals; there are short intervals during which signals are not locally coupled and during which phase difference should not be estimated, the shortest lasting $30 \mathrm{~s}$; phase difference varies non-linearly with time; the amplitude modulation first undergoes stepwise changes and then recovery toward higher values following a non-linear time-course; noise is added; finally, all these patterns imply simultaneous amplitude and frequency modulation of the signals. Results were obtained over 50 realizations and are shown in Figure 1.6, where the time-course of $\gamma_{0}(t), \theta_{0}(t)$ and $\alpha_{0}(t)$ is represented by a red-bold line, while estimates obtained through time-frequency analysis in represented in gray. Signals $x(t)$ and $y(t)$ are uncoupled during two intervals where $\gamma_{0}(t)=0$, their phase difference changes as a sinusoids and the amplitude modulation presents slow and abrupt non-stationarities. Results demonstrate that the presented framework provides an accurate estimation of the changes in the signal properties and their dynamic interactions.

\subsubsection{Time-frequency phase locking}

The time-frequency representation of phase differences offers the possibility of estimating the degree of phase-locking between different signals $[60,61,56,57,63,64]$. The phase locking is used to assess whether a given stimulus provokes similar changes in the time-frequency phase difference spectrum. Its 

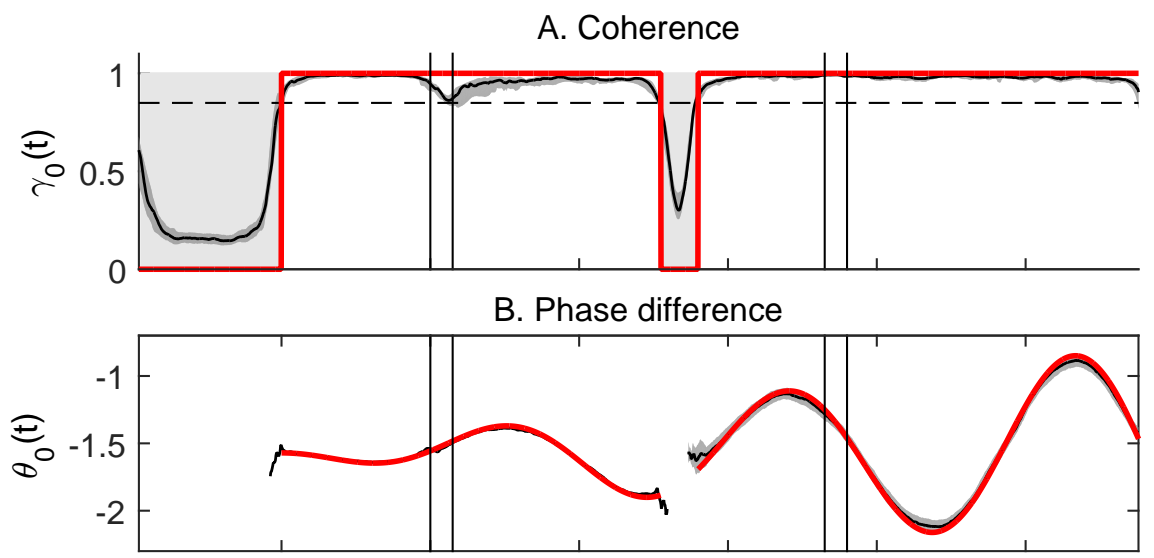

C. Amlitude modulation

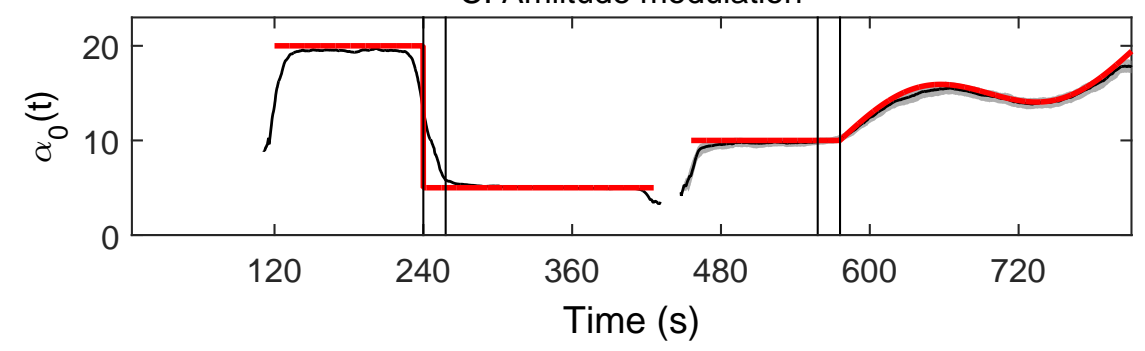

Figure 1.6: Simulation: Time-course of (A) Coherence, (B) Phase differences, (C) Amplitude modulation. Reference values $\gamma_{0}(t), \theta_{0}(t)$ and $\alpha_{0}(t)$ described in equations (1.33) are represented in bold red lines. Estimates are represented as median (black line) and interquartile range (gray region). Vertical lines mark supine positions and head-up tilt. Light gray areas represent the intervals during which signals are uncoupled. The horizontal dashed line in (A) represents the threshold for statistical significance.

time-frequency representation is [65]:

$$
\Psi(t, f)=\left|\frac{1}{L} \sum_{i=1}^{L} e^{j 2 \pi \Theta_{\mathrm{i}}(t, f)}\right|=\left|\frac{1}{L} \sum_{i=1}^{L} \frac{S_{\mathrm{xy}, \mathrm{i}}(t, f)}{\left|S_{\mathrm{x}, \mathrm{i}}(t, f)\right|}\right|
$$

By construction, $\Psi(t, f)$ is bounded between 0 and $1, \Psi(t, f) \in[0,1]$, being $\Psi(t, f)=1$ if $\Theta_{\mathrm{i}}(t, f)$ does not vary across different realizations $i$, and being $\Psi(t, f)=0$ if $\Theta_{\mathrm{i}}(t, f)$ is characterized by a zero mean random distribution across $i$. Indeed, if for a given point $\left(t_{0}, f_{0}\right)$, the complex terms in the summation in (1.35) maintain similar phase for all $i, \Psi\left(t_{0}, f_{0}\right) \approx 1$.

Therefore, the phase locking measures the degree of similarity of the time-frequency structure of a group of signals, by pairwise comparing their time-frequency phase differences $\Theta_{\mathrm{i}}(t, f)$.

\subsubsection{Time-frequency partial spectrum}

The estimation of a time-frequency partial spectrum is interesting in the analysis of systems that can be modeled as the sum of two single-input/output models where inputs $u(t)$ and $v(t)$ are uncorrelated (see Figure 1.7):

$$
y(t)=x_{1}(t)+x_{2}(t)=h_{1}(t) * u(t)+h_{2}(t) * v(t)
$$

where $*$ represents convolution with time-varying transfer functions $h_{1}(t)$ and $h_{2}(t)$.

In many applications, one needs to decompose the TF spectrum of $y(t)$ into a part related to $x_{1}(t)$ and another related to $x_{2}(t)$. This section describes how to obtain such a decomposition when only te output $y(t)$ and one of the inputs, i.e. either $u(t)$ or $v(t)$, are known. In the time-frequency domain, 


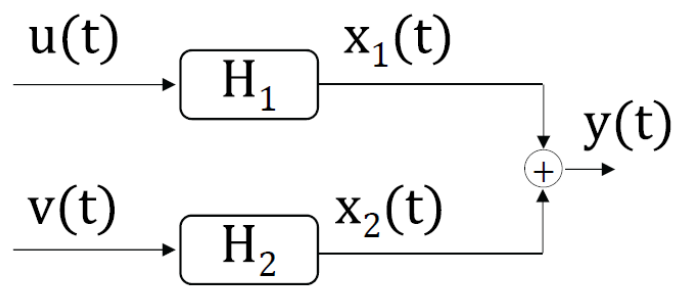

Figure 1.7: A multiple-input single-output model. Inputs $u(t)$ and $v(t)$ are uncorrelated.

input/output auto spectrum and input/output cross spectrum relations are:

$$
\begin{aligned}
H_{1}(t, f) & =\frac{S_{\mathrm{u}, 1}(t, f)}{S_{\mathrm{u}, \mathrm{u}}(t, f)} \\
\left|H_{1}(t, f)\right|^{2} & =\frac{S_{1,1}(t, f)}{S_{\mathrm{u}, \mathrm{u}}(t, f)}
\end{aligned}
$$

Taking into account that the inputs are uncorrelated, the TF spectrum of the output is [66]:

$$
S_{\mathrm{y}, \mathrm{y}}(t, f)=\left|H_{1}\right|^{2} S_{1,1}(t, f)+\left|H_{2}\right|^{2} S_{2,2}(t, f)
$$

while TF coherence between $y(t)$ and input $u(t)$ is:

$$
\gamma_{\mathrm{y}, \mathrm{u}}^{2}(t, f)=\frac{1}{1+S_{2,2}(t, f) / S_{1,1}(t, f)}
$$

where $S_{1,1}(t, f)$ and $S_{2,2}(t, f)$ are auto spectra of $x_{1}(t)$ and $x_{2}(t)$. In this formulation, $x_{2}(t)$ is considered a noise term uncorrealted with input $u(t)$. Similarly, the TF coherence between $y(t)$ and input $v(t)$ is

$$
\gamma_{\mathrm{y}, \mathrm{v}}^{2}(t, f)=\frac{1}{1+S_{1,1}(t, f) / S_{2,2}(t, f)}
$$

where $x_{1}(t)$ is considered a noise term uncorrealted with input $v(t)$.

The TF coherence function can be used to separate the output spectrum into two partial spectra: the coherent output spectrum and the partial (or conditioned) spectrum [66]:

$$
\begin{aligned}
& S_{y: 1}(t, f)=S_{1,1}(t, f)=\gamma_{y, u}^{2}(t, f) S_{y, y}(t, f)=\left(1-\gamma_{y, v}^{2}(t, f)\right) S_{y, y} \\
& S_{y: 2}(t, f)=S_{2,2}(t, f)=\gamma_{y, v}^{2}(t, f) S_{y, y}(t, f)=\left(1-\gamma_{y, u}^{2}(t, f)\right) S_{y, y}
\end{aligned}
$$

Note that $S_{1,1}(t, f)$ can be calculated from output $y(t)$ and input $u(t)$ even if $x_{1}(t)$ cannot be measured. Similarly, $S_{2,2}(t, f)$ can also be estimated from $y(t)$ and input $u(t)$ without measuring $x_{2}(t)$. Definitions (1.42)-(1.43) show that a partial TF spectrum is obtained from the original spectrum by using the TFC function as a TF filter that reduces the amplitude of the original spectrum by a factor proportional to the TFC. In regions where the TFC is equal to one, the TF partial spectrum is equal to zero. Whereas in regions where the TFC is zero, the original and the partial spectra will have the same amplitude. This approach has been used to estimate changes in the variability of the QT interval unrelated with heart rate variability, by using records of QT and RR intervals [67].

\subsection{Example of analysis of cardiovascular signals}

The time-frequency framework described in this chapter has been applied to the study of cardiovascular signals and their dynamic interactions [14, 2, 46, 65], and references to specific studies have been given and briefly discussed in the previous sections. In this section, cardiovascular signals from one healthy 
volunteer undergoing a head-up tilt test are studied as a representative example. This test includes three phases: early supine position $(4 \mathrm{~min})$, head-up tilt at $70^{\circ}(5 \mathrm{~min})$ and late supine position $(4 \mathrm{~min})$ [2]. The RR interval was measured from the surface ECG, systolic arterial blood pressure was measured non-invasively from the finger using a Finometer device, and respiration was measured as chest movement using a thoracic belt. Results shown in Figure 1.1 and Figure 1.6 are from the same subject. Time-frequency analysis was performed using the kernel function described in (1.26). The time and frequency resolutions, measured as the full-width at half maximum of the kernel function evaluated at its center [14], were equal to $11 \mathrm{~s}$ and $0.039 \mathrm{~Hz}$, respectively. A statistical test to estimate the threshold for significant coupling was conducted using white Gaussian noise as uncoupled surrogate data. The threshold associated with a $5 \%$ significance level was equal to 0.849 .

Figure 1.8 shows the time-frequency spectra of RR variability (RRV), systolic arterial pressure variability (SAPV) and the respiratory signal (R). RRV and SAPV show spectral components in both the LF (0.04-0.15 Hz) and HF $(0.15-0.40 \mathrm{~Hz})$ bands, while the spectral power of the respiratory signal is in the HF band only. Interestingly, both amplitudes and frequencies of these signals clearly change during the test. The time-frequency resolution of these representations allows to track these changes. In particular, subtle changes in the instantaneous frequency of the respiratory oscillations can be followed accurately. Time-frequency coherence (TFC) and time-frequency phase difference (TFPD) between each pair of signals is also reported. Regions of the time-frequency domain where the coherence between two signals is statistically significant are encircled by a black line. These time-frequency coherence representations demonstrate that the three signals are strongly locally coupled. Significant level of coupling between RRV and SAPV is found in both LF and HF, whereas respiration is strongly coupled with only HF components of RRV and SAPV. The coupling in HF is maintained during the entire test, demonstrating that respiratory oscillations in RRV and SAPV are persistent. Whereas the coupling in the LF band is intermittent.

The inspection of the TFPD representations reveals that the phase difference between coupled oscillations in these cardiovascular signals is affected by head-up tilt. A clear and sudden change in the phase difference after the onset of head-up tilt is registered between RRV and SAPV, RRV and R, and between SAPV and R. In the late supine position, baseline values are restored, suggesting that head-up tilt is provoking a shift in the degree of temporal synchronization of respiratory-related oscillations within the cardiovascular system.

Figure 1.9 shows the time-frequency partial spectra of RRV (upper panel) and SAPV (lower panel) after removing the components in synchrony with respiration. These representations have been obtained using the definition (1.42) and the kernel (1.26). The comparison between these representations and the original RRV and SAPV spectra shown in Fig. 1.8 suggests that the HF components of both signals are almost entirely due to respiration. The residual components still present in the TF spectra are mainly in the LF band. Black lines encircle the TF regions where TFC with respiration is statistically significant. As expected, in these regions the amplitude of the TF partial spectra is particularly low.

\subsection{Final considerations}

In this chapter, we have described a framework for the assessment of the time-frequency structure of non-stationary signals and their dynamic interactions. We have used simulated data to demonstrate its reliability and we have provided examples based on cardiovascular signals recorded during a tilttable test, a procedure that quickly modifies arterial blood pressure and activates a neural response (the baroreflex) to restore baseline values. The approach described in this chapter has been proven to be 
robust and accurate $[14,2,46]$ and it is certainly a useful tool to track the cardiovascular response to different stimuli, such as emotions [68], orthostatic stress [2], exercise stress test [69] etc., and to detect subtle and transient dynamics such as repolarization alternans [70].

In recent years, different methods to estimate time-varying features of non-stationary signals have been proposed. Most of them are based on parametric autoregressive modeling [4, 71, 72, 73]. The performance of these methods is related to their capability of capturing signal dynamics and, in extremely non-stationary conditions, they have been observed to perform less accurately than non-parametric methods $[45,74]$.

Time-varying estimators based on non-parametric methods have the advantage of not requiring any assumption on the TF structure of the signals and are relatively easy to estimate. Beside those described in this chapter, others have been used in biomedical applications. These include the time-scale coherence [75] based on the continuous wavelet transform, which has been proposed to study cardiovascular dynamics $[76,77,50]$ as well as neural connectivity $[63,78,79,80]$. These methods are characterized by a non-homogeneous TF resolution and are suited for signals presenting high frequency components of short duration and low frequency components of long duration. In the late Nineties, estimators of time-frequency coherence based on multitaper spectrogram [81, 82] have also been proposed to study the coupling between neuronal signals [83] and atrial fibrillation [84].

The main limitation of the time-frequency framework described in this chapter is that it cannot be used to infer causality or to assess the direction of the coupling. In this case, other approaches, mainly parametric [85], should be used.

\section{Acknowledgments}

The Matlab code to conduct the analysis and create the figures shown in this chapter is are available at $[86]$. 


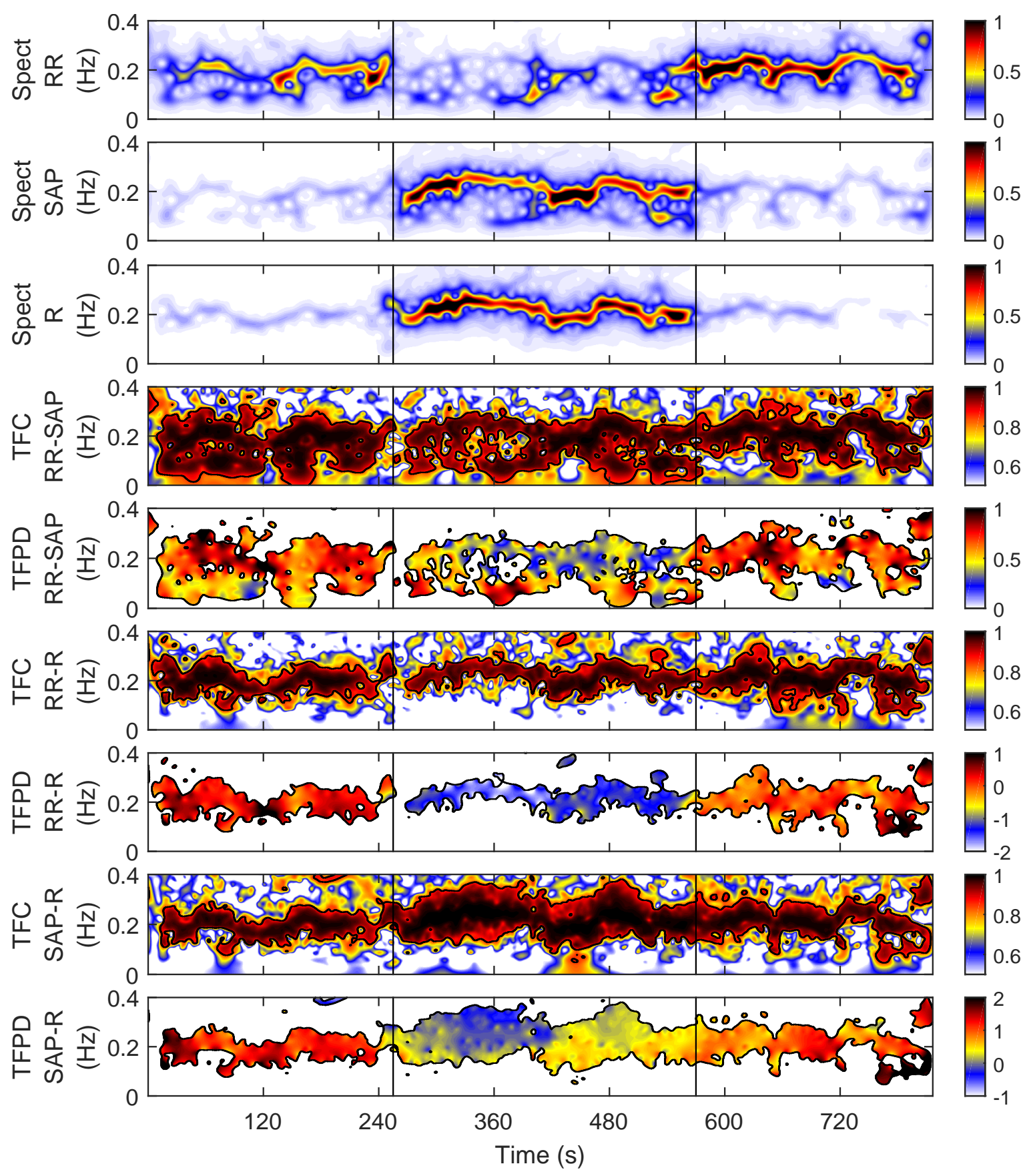

Figure 1.8: Time-frequency analysis of RR interval variability (RRV), systolic arterial blood pressure variability $(\mathrm{SAPV})$ and respiration $(\mathrm{R})$ during head-up tilt table test. Vertical lines separate the early supine phase $(4 \mathrm{~min})$, from the head-up tilt phase $(5 \mathrm{~min})$, and the late supine phase $(4 \mathrm{~min})$. From top to bottom: The first three panels show the time-frequency spectra of RRV, SAPV and R, respectively. From the forth panels onwards, figures show the time-frequency coherence and phase difference of RRV and SAPV, RRV and R, and finally of SAPV and R. Black lines in the coherence and phase differences panels encircle the regions where coherence is statistically significant $(\alpha<5 \%)$. Time-frequency phase is shown only in these regions. Colors represent arbitrary units for the TF spectra, normalized units for the TFC, and radians for the TFPD. 

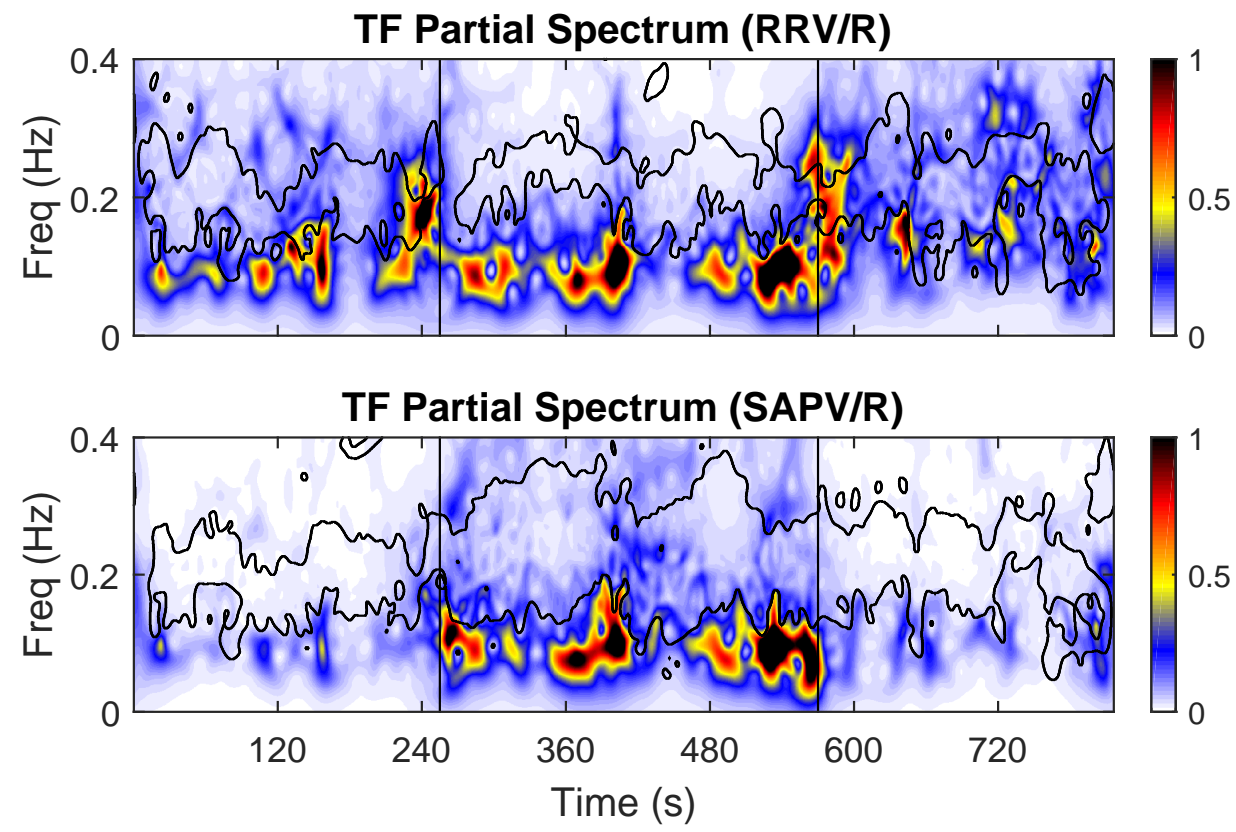

Figure 1.9: Time-frequency partial spectra of RR interval variability (RRV) and systolic arterial pressure variability (SAPV) unrelated to respiration during head-up tilt table test. Vertical lines separate the early supine phase $(4 \mathrm{~min})$, from the head-up tilt phase $(5 \mathrm{~min})$, and the late supine phase $(4 \mathrm{~min})$. These representations greatly differ from the original TF spectra shown in Figure 1.8. In particular, HF components strongly coupled with respiration have been almost entirely removed. The black lines in these panels encircle the regions where coherence with respiration is statistically significant $(\alpha<5 \%)$. Note that, as expected, inside these regions the time-frequency partial spectra show very little power. In the TF spectra, colors represent arbitrary units. 


\section{Bibliography}

[1] P. Borgnat, P. Flandrin, P. Honeine, C. Richard, and J. Xiao, "Testing stationarity with surrogates: A time-frequency approach," IEEE Trans. Signal Process., vol. 58, no. 7, pp. 3459-3470, 2010.

[2] M. Orini, P. Laguna, L. T. Mainardi, and R. Bailón, "Assessment of the dynamic interactions between heart rate and arterial pressure by the cross time-frequency analysis.," Physiol. Meas., vol. 33, pp. 315-331, Mar 2012.

[3] L. T. Mainardi, A. M. Bianchi, G. Baselli, and S. Cerutti, "Pole-tracking algorithms for the extraction of time-variant heart rate variability spectral parameters," Biomedical Engineering, IEEE Transactions on, vol. 42, no. 3, pp. 250-259, 1995. ID: 1.

[4] L. T. Mainardi, A. M. Bianchi, R. Furlan, S. Piazza, R. Barbieri, V. di Virgilio, A. Malliani, and S. Cerutti, "Multivariate time-variant identification of cardiovascular variability signals: a beat-tobeat spectral parameter estimation in vasovagal syncope," Biomedical Engineering, IEEE Transactions on, vol. 44, pp. 978-989, Oct. 1997.

[5] R. Barbieri and E. Brown, "Analysis of heartbeat dynamics by point process adaptive filtering," Biomedical Engineering, IEEE Transactions, vol. 53, no. 1, pp. 4-12, 2006.

[6] P. Welch, "The use of fast fourier transform for the estimation of power spectra: A method based on time averaging over short, modified periodograms," IEEE Trans. Audio Electroacoust., vol. 15, pp. 70-73, Jun 1967.

[7] D. J. Thomson, "Spectrum estimation and harmonic analysis," Proc. IEEE, vol. 70, no. 9, pp. 1055$1096,1982$.

[8] S. Akselrod, D. Gordon, F. A. Ubel, D. C. Shannon, A. C. Barger, and R. J. Cohen, "Power spectrum analysis of heart rate fluctuations: a quantitative probe of beat-to-beat cardiovascular control," Science, vol. 213, pp. 220-222, 1981.

[9] J. P. Saul, Y. Arai, R. D. Berger, L. S. Lilly, W. S. Colucci, and R. J. Cohen, "Assessment of autonomic regulation in chronic congestive heart failure by heart rate spectral analysis," The American journal of cardiology, vol. 61, no. 15, pp. 1292-1299, 1988.

[10] Task Force of the European Society of Cardiology the North American Society of Pacing, "Heart rate variability : Standards of measurement, physiological interpretation, and clinical use," Circulation, vol. 93, no. 5, pp. 1043-1065, 1996.

[11] N. Montano, T. G. Ruscone, A. Porta, F. Lombardi, M. Pagani, and A. Malliani, "Power spectrum analysis of heart rate variability to assess the changes in sympathovagal balance during graded orthostatic tilt," Circulation, vol. 90, pp. 1826-1831, 1994. 
[12] A. Malliani, M. Pagani, F. Lombardi, and S. Cerutti, "Cardiovascular neural regulation explored in the frequency domain," Circulation, vol. 84, pp. 482-492, 1991.

[13] G. Piccirillo, M. Ogawa, J. Song, V. Chong, B. Joung, S. Han, D. Magra, L. Chen, S.-F. Lin, and P.-S. Chen, "Power spectral analysis of heart rate variability and autonomic nervous system activity measured directly in healthy dogs and dogs with tachycardia-induced heart failure," vol. 6, no. 4, pp. 546-552, 2009 .

[14] M. Orini, R. Bailon, L. T. Mainardi, P. Laguna, and P. Flandrin, "Characterization of dynamic interactions between cardiovascular signals by time-frequency coherence.," IEEE Trans. Biomed. Eng., vol. 59, pp. 663-673, Mar 2012.

[15] P. Grossman and E. W. Taylor, "Toward understanding respiratory sinus arrhythmia: Relations to cardiac vagal tone, evolution and biobehavioral functions," Biol. Psychol., vol. 74, pp. 263-285, Feb. 2007.

[16] D. L. Eckberg, "Point:counterpoint: respiratory sinus arrhythmia is due to a central mechanism vs. respiratory sinus arrhythmia is due to the baroreflex mechanism.," J. Appl. Physiol., vol. 106, pp. 1740-2; discussion 1744, May 2009.

[17] C. Julien, "The enigma of mayer waves: Facts and models.," Cardiovasc. Res., vol. 70, pp. 12-21, Apr 2006.

[18] D. L. Eckberg, "Sympathovagal balance : A critical appraisal," Circulation, vol. 96, no. 9, pp. 3224$3232,1997$.

[19] A. Malliani, M. Pagani, N. Montano, and G. S. Mela, "Sympathovagal balance: A reappraisal," Circulation, vol. 98, no. 23, pp. 2640-2643, 1998.

[20] A. Malliani, C. Julien, G. E. Billman, S. Cerutti, M. F. Piepoli, L. Bernardi, P. Sleight, M. A. Cohen, C. O. Tan, D. Laude, M. Elstad, K. Toska, J. M. Evans, and D. L. Eckberg, "Cardiovascular variability is/is not an index of autonomic control of circulation," J. Appl. Physiol., vol. 101, no. 2, pp. 684-688, 2006 .

[21] P. Flandrin, Time-Frequency/Time-Scale Analysis. Academic Press, New York, 1999.

[22] L. Cohen, Time-frequency analysis, vol. 778. Prentice hall, 1995.

[23] F. Hlawatsch and F. Auger, eds., Time-frequency analysis, concepts and methods. wilew, 2008.

[24] L. Cohen, "Time-frequency distributions-a review," Proc. IEEE, vol. 77, pp. 941-981, July 1989.

[25] F. Hlawatsch and G. F. Boudreaux-Bartels, "Linear and quadratic time-frequency signal representations," Signal Processing Magazine, IEEE, vol. 9, pp. 21-67, April 1992.

[26] F. Auger, P. Flandrin, G. P., and L. Olivier, "Time-frequency toolbox," tech. rep., http://www.nongnu.org/tftb/.

[27] Flandrin, Ambiguity Function, ch. Ambiguity Function, pp. 160-167. Elsevier, 2003.

[28] F. Auger and r. Chassande-Mottin, Quadratic Time-Frequency Analysis I: Cohen's Class, pp. 131163. ISTE, 2010.

[29] J. Jeong and W. J. Williams, "Kernel design for reduced interference distributions," IEEE Trans. Signal Process., vol. 40, no. 2, pp. 402-412, 1992. 
[30] R. G. Baraniuk and D. L. Jones, "A signal-dependent time-frequency representation: optimal kernel design," Signal Processing, IEEE Transactions on, vol. 41, pp. 1589-1602, April 1993.

[31] R. G. Baraniuk and D. L. Jones, "A signal-dependent time-frequency representation: fast algorithm for optimal kernel design," IEEE Trans. Signal Process., vol. 42, no. 1, pp. 134-146, 1994.

[32] G. S. Cunningham and W. J. Williams, "Kernel decomposition of time-frequency distributions," IEEE Trans. Signal Process., vol. 42, no. 6, pp. 1425-1442, 1994.

[33] A. Costa and G. Boudreau-Bartels, "Design of time-frequency representations using a multiform, tiltable exponential kernel," Signal Processing, IEEE Transactions on, vol. 43, pp. 2283-2301, Oct 1995.

[34] G. R. Arce and S. R. Hasan, "Elimination of interference terms of the discrete wigner distribution using nonlinear filtering," IEEE Trans. Signal Process., vol. 48, no. 8, pp. 2321-2331, 2000.

[35] S. Aviyente and W. J. Williams, "Multitaper marginal time-frequency distributions," Signal Process., vol. 86, no. 2, pp. 279-295, 2006.

[36] P. Wahlberg and M. Hansson, "Kernels and multiple windows for estimation of the wigner-ville spectrum of gaussian locally stationary processes," IEEE Trans. Signal Process., vol. 55, no. 1, pp. 73-84, 2007.

[37] F. Hlawatsch, "Interference terms in the wigner distribution," in Digital signal processing (V. Cappellini and A. Constantinides, eds.), vol. 84, pp. 363-267, 1984.

[38] F. Hlawatsch and P. Flandrin, The Wigner Distribution - theory and applications in signal processing, ch. The interference structure of the Wigner distribution and related time-frequency signal representations, pp. 59-113. Elsevier, 1997.

[39] P. Flandrin, "Some features of time-frequency representations of multicomponent signals," Acoustics, Speech, and Signal Processing, IEEE International Conference on ICASSP '84., vol. 9, pp. 266-269, 1984.

[40] M. Orini, R. Bailón, L. Mainardi, A. Mincholé, and P. Laguna, "Continuous quantification of spectral coherence using quadratic time-frequency distributions: error analysis and application," Internat. Conf. Computers in Cardiology, pp. 681-684, 2009.

[41] W. Martin and P. Flandrin, "Wigner-ville spectral analysis of nonstationary processes," Acoustics, Speech and Signal Processing, IEEE Transactions on, vol. 33, no. 6, pp. 1461-1470, 1985. ID: 1.

[42] A. Janssen and T. Claasen, "On positivity of time-frequency distributions," IEEE Trans. Acoust., Speech, Signal Process., vol. 33, pp. 1029-1032, Aug 1985.

[43] G. Matz and F. Hlawatsch, "Nonstationary spectral analysis based on time-frequency operator symbols and underspread approximations," Information Theory, IEEE Transactions on, vol. 52, no. 3, pp. 1067-1086, 2006. ID: 1.

[44] L. T. Mainardi, "On the quantification of heart rate variability spectral parameters using timefrequency and time-varying methods," Philosophical transactions.Series A, Mathematical, physical, and engineering sciences, vol. 367, no. 1887, pp. 255-275, 2009.

[45] S. Pola, A. Macerata, M. Emdin, and C. Marchesi, "Estimation of the power spectral density in nonstationary cardiovascular time series: assessing the role of the time-frequency representations (tfr).," IEEE Trans. Biomed. Eng., vol. 43, pp. 46-59, Jan 1996. 
[46] M. Orini, R. Bailon, P. Laguna, L. Mainardi, and R. Barbieri, "A multivariate time-frequency method to characterize the influence of respiration over heart period and arterial pressure," EURASIP Journal on Advances in Signal Processing, vol. 2012, no. 1, p. 214, 2012.

[47] G. C. Carter, "Coherence and time delay estimation," Proc. IEEE, vol. 75, pp. 236-255, Feb. 1987.

[48] M. Di Rienzo, G. Parati, A. Radaelli, and P. Castiglioni, "Baroreflex contribution to blood pressure and heart rate oscillations: time scales, time-variant characteristics and nonlinearities," Philosophical Transactions of the Royal Society A: Mathematical,Physical and Engineering Sciences, vol. 367, pp. 1301-1318, Apr. 2009.

[49] M. Orini, L. T. Mainardi, E. Gil, P. Laguna, and R. Bailon, "Dynamic assessment of spontaneous baroreflex sensitivity by means of time-frequency analysis using either rr or pulse interval variability.," Conf Proc IEEE Eng Med Biol Soc, vol. 1, pp. 1630-1633, 2010.

[50] K. Keissar, R. Maestri, G. D. Pinna, M. T. L. Rovere, and O. Gilad, "Non-invasive baroreflex sensitivity assessment using wavelet transfer function-based time-frequency analysis.," Physiol. Meas., vol. 31, pp. 1021-1036, Jul 2010.

[51] E. Gil, M. Orini, R. Bailon, J. M. Vergara, L. Mainardi, and P. Laguna, "Photoplethysmography pulse rate variability as a surrogate measurement of heart rate variability during non-stationary conditions," Physiol. Meas., vol. 31, no. 9, pp. 1271-1290, 2010.

[52] G. Matz and F. Hlawatsch, "Time-frequency coherence analysis of nonstationary random processes," in Proc. Tenth IEEE Workshop Statistical Signal and Array Processing, pp. 554-558, 2000.

[53] L. B. White and B. Boashash, "Cross spectral analysis of nonstationary processes," Information Theory, IEEE Transactions on, vol. 36, pp. 830-835, July 1990.

[54] L. Faes, G. D. Pinna, A. Porta, R. Maestri, and G. Nollo, "Surrogate data analysis for assessing the significance of the coherence function," IEEE Trans. Biomed. Eng., vol. 51, pp. 1156-1166, July 2004 .

[55] T. Schreiber and A. Schmitz, "Surrogate time series," Physica D: Nonlinear Phenomena, vol. 142, no. $3-4$, pp. $346-382,2000$.

[56] J. P. Lachaux, E. Rodriguez, J. Martinerie, and F. J. Varela, "Measuring phase synchrony in brain signals.," Hum. Brain Mapp., vol. 8, no. 4, pp. 194-208, 1999.

[57] J. Lachaux, E. Rodriguez, M. L. V. Quyen, A. Lutzand, J. Martinerie, and F. Varela, "Studying single-trials of phase-synchronous activity in the brain," Int J Bifurcat Chaos, vol. 10, pp. 2439-39, 2000 .

[58] M. L. V. Quyen, J. Foucher, J. Lachaux, E. Rodriguez, A. Lutz, J. Martinerie, and F. J. Varela, "Comparison of hilbert transform and wavelet methods for the analysis of neuronal synchrony.," $J$. Neurosci. Methods, vol. 111, pp. 83-98, Oct 2001.

[59] D. Rudrauf, A. Douiri, C. Kovach, J.-P. Lachaux, D. Cosmelli, M. Chavez, C. Adam, B. Renault, J. Martinerie, and M. L. V. Quyen, "Frequency flows and the time-frequency dynamics of multivariate phase synchronization in brain signals.," Neuroimage, vol. 31, pp. 209-227, May 2006.

[60] S. Aviyente, E. M. Bernat, W. S. Evans, and S. R. Sponheim, "A phase synchrony measure for quantifying dynamic functional integration in the brain," Hum. Brain Mapp., vol. 32, no. 1, pp. 8093, 2011. 
[61] S. Aviyente, W. S. Evans, E. M. Bernat, and S. Sponheim, "A time-varying phase coherence measure for quantifying functional integration in the brain," in Proc. IEEE International Conference on Acoustics, Speech and Signal Processing ICASSP 2007, vol. 4, pp. IV-1169-IV-1172, 15-20 April 2007.

[62] Y. Shin, D. Gobert, S.-H. Sung, E. J. Powers, and J. B. Park, "Application of cross time-frequency analysis to postural sway behavior: the effects of aging and visual systems," IEEE Trans. Biomed. Eng., vol. 52, pp. 859-868, May 2005. Time-frequency phase cross time-frequency analysis.

[63] J.-P. Lachaux, A. Lutz, D. Rudrauf, D. Cosmelli, M. L. V. Quyen, J. Martinerie, and F. Varela, "Estimating the time-course of coherence between single-trial brain signals: an introduction to wavelet coherence.," Neurophysiol. Clin., vol. 32, pp. 157-174, Jun 2002.

[64] M. Orini, P. Laguna, L. Mainardi, and R. Bailón, "Characterization of the dynamic interactions between cardiovascular signals by cross time-frequency analysis: phase differences, time delay and phase locking," Int. Conf. on numerical method in engineering, 2011.

[65] M. Orini, R. Bailon, L. T. Mainardi, and P. Laguna, "Time-frequency phase differences and phase locking to characterize dynamic interactions between cardiovascular signals," Conf Proc IEEE Eng Med Biol Soc, vol. 1.

[66] J. S. Bendat and A. G. Piersol, "Multiple-input/output relationships," in Random Data, pp. 201247, John Wiley \& Sons, Inc., 2012.

[67] M. Orini, P. Taggart, and P. D. Lambiase, "A multivariate time-frequency approach for tracking QT variability changes unrelated to heart rate variability," Conf Proc IEEE Eng Med Biol Soc, vol. Submitted, pp. - , 2016.

[68] M. Orini, R. Bailón, R. Enk, S. Koelsch, L. Mainardi, and P. Laguna, "A method for continuously assessing the autonomic response to music-induced emotions through hrv analysis.," Med. Biol. Eng. Comput., vol. 48, pp. 423-433, May 2010.

[69]

[70] M. Orini, B. Hanson, P. Taggart, and P. Lambiase, "Detection of transient, regional cardiac repolarization alternans by time-frequency analysis of synthetic electrograms," Engineering in Medicine and Biology Society (EMBC), 2013 35th Annual International Conference of the IEEE, pp. 3773-3776, 2013.

[71] H. Zhao, S. Lu, R. Zou, K. Ju, and K. H. Chon, "Estimation of time-varying coherence function using time-varying transfer functions.," Ann. Biomed. Eng., vol. 33, pp. 1582-1594, Nov 2005.

[72] H. Zhao, W. A. Cupples, K. H. Ju, and K. H. Chon, "Time-varying causal coherence function and its application to renal blood pressure and blood flow data," Biomedical Engineering, IEEE Transactions on, vol. 54, pp. 2142-2150, Dec. 2007.

[73] Z. Chen, P. Purdon, G. Harrell, E. Pierce, J. Walsh, E. Brown, and R. Barbieri, "Dynamic assessment of baroreflex control of heart rate during induction of propofol anesthesia using a point process method," Ann. Biomed. Eng., vol. 39, pp. 260-276, 2011. 10.1007/s10439-010-0179-z.

[74] M. Orini, R. Bailon, P. Laguna, and L. T. Mainardi, "Modeling and estimation of time-varying heart rate variability during stress test by parametric and non parametric analysis," in Proc. Computers in Cardiology, pp. 29-32, Sept. 30 2007-Oct. 32007. 
[75] A. Grinsted, J. C. Moore, and S. Jevrejeva, "Application of the cross wavelet transform and wavelet coherence to geophysical time series," Nonlinear Processes in Geophysics, vol. 11, pp. 561-566, 2004.

[76] C. Gallet, B. Chapuis, C. Barrès, and C. Julien, "Time-frequency analysis of the baroreflex control of renal sympathetic nerve activity in the rat," J. Neurosci. Methods, vol. 198, no. 2, pp. 336 - 343, 2011.

[77] K. Keissar, L. R. Davrath, and S. Akselrod, "Coherence analysis between respiration and heart rate variability using continuous wavelet transform," Philosophical Transactions of the Royal Society A: Mathematical, Physical and Engineering Sciences, vol. 367, no. 1892, pp. 1393-1406, 2009.

[78] Y. Zhan, D. Halliday, P. Jiang, X. Liu, and J. Feng, "Detecting time-dependent coherence between non-stationary electrophysiological signals-a combined statistical and time-frequency approach.," $J$. Neurosci. Methods, vol. 156, pp. 322-332, Sep 2006.

[79] J. Bigot, M. Longcamp, D. M. F, and D. Amarantini, "A new statistical test based on the wavelet cross-spectrum to detect time-frequency dependence between non-stationary signals: Application to the analysis of cortico-muscular interactions.," Neuroimage, Jan 2011.

[80] J. S. Brittain, D. M. Halliday, B. A. Conway, and J. B. Nielsen, "Single-trial multiwavelet coherence in application to neurophysiological time series," IEEE Trans. Biomed. Eng., vol. 54, pp. 854-862, May 2007.

[81] J. Xiao and P. Flandrin, "Multitaper time-frequency reassignment for nonstationary spectrum estimation and chirp enhancement," IEEE Trans. Signal Process., vol. 55, pp. 2851-2860, June 2007.

[82] D. J. Thomson, "Jackknifing multitaper spectrum estimates," IEEE Signal Process. Mag., vol. 24, pp. 20-30, 072007.

[83] Y. Xu, S. Haykin, and R. J. Racine, "Multiple window time-frequency distribution and coherence of eeg using slepian sequences and hermite functions," IEEE Trans. Biomed. Eng., vol. 46, no. 7, pp. 861-866, 1999 .

[84] E. G. Lovett and K. M. Ropella, "Time-frequency coherence analysis of atrial fibrillation termination during procainamide administration.," Ann. Biomed. Eng., vol. 25, no. 6, pp. 975-984, 1997. multitaper, spectrogram,coherence.

[85] L. Faes and G. Nollo, Biomedical Engineering, Trends in Electronics, Communications and Software, ch. Multivariate Frequency Domain Analysis of Causal Interactions in Physiological Time Series. 2011.

[86] "http://www.micheleorini.com/matlab-code/." 